\title{
Genomic analysis of Leptospira interrogans serovar Paidjan and Dadas isolates from carrier dogs and comparative genomic analysis to detect genes under positive selection
}

\author{
Alongkorn Kurilung ${ }^{1}$, Chantisa Keeratipusana ${ }^{2}$, Prapat Suriyaphol ${ }^{2}$, David J. Hampson ${ }^{3}$ and Nuvee Prapasarakul ${ }^{1,4^{*}}$ (1)
}

\begin{abstract}
Background: Leptospirosis is an emerging infectious disease worldwide that can cause high morbidity and mortality rates in humans and animals. The causative spirochetes have reservoirs in mammalian hosts, but there has been limited analysis of the genomes of isolates recovered from animals. The aims of this study were to characterize genomic features of two Leptospira interrogans strains recently isolated from asymptomatic dogs in Thailand (strains CUDO5 and CDUO8), and to perform comparative genome analyses with other strains. Molecular adaptive evolution in L. interrogans as signaled by positive selection also was analyzed.
\end{abstract}

Results: Whole genome sequence analysis revealed that strains CUDO5 and CUDO8 had genome sizes of approximately 4.9 Mbp with 35.1\% GC contents. Using monoclonal antibodies, strains CUDO5 and CUDO8 were identified as serovars Paidjan and Dadas, respectively. These strains harbored genes known to be associated with acute and chronic infections. Using Single Nucleotide Polymorphisms phylogeny (SNPs) with 97 L. interrogans strains, CUDO5 and CUDO8 had closest genetic relatedness with each other. Nevertheless, the serovar determinant region (rfb locus) showed variations in the genes encoding sugar biosynthesis. Amongst 13 representative $L$. interrogans strains examined for molecular adaptive evolution through positive selection under the site-model of Phylogenetic Analysis of Maximum Likelihood, genes responsible for iron acquisition (tlyA and $h b p A$ ), motility (fliN2, flgK, and flhB) and thermal adaptation (IpxD1) were under increased selective pressure.

Conclusions: L. interrogans serovar Paidjan strain CUDO5 and serovar Dadas strain CUDO8 had close genetic relatedness as analyzed by SNPs phylogeny. They contained genes with established roles in acute and chronic leptospirosis. The rfb locus in both serovars showed gene variation associated with sugar biosynthesis. Positive selection analysis indicated that genes encoding factors involved in motility, temperature adaptation, and iron acquisition were under strong positive selection in L. interrogans. These may be associated with adaptation in the early stages of infection.

Keywords: Genome, Leptospira interrogans, Serovar Paidjan, Serovar Dadas, Dog, Positive selection

\footnotetext{
* Correspondence: nuvee.p@chula.ac.th

${ }^{1}$ Department of Microbiology, Faculty of Veterinary Science, Chulalongkorn University, Bangkok, Thailand

${ }^{4}$ Diagnosis and Monitoring of Animal Pathogens Research Unit, Department

of Microbiology, Faculty of Veterinary Science, Chulalongkorn University,

Bangkok, Thailand

Full list of author information is available at the end of the article
} 


\section{Background}

Gram-negative spirochetes of the genus Leptospira cause the disease Leptospirosis, which is one of the most important zoonotic infectious conditions worldwide, including in Thailand [1, 2]. Pathogenic Leptospira strains can infect most mammals and produce a wide range of clinical manifestations varying from acute life-threatening disease to chronic asymptomatic forms [3]. In humans, leptospirosis is estimated to have a worldwide annual average morbidity of 1.03 million cases with a mortality of 58,900 cases, and these figures are poised to increase due to climate change [4]. Asymptomatic animals that are carrying leptospires in the convoluted tubules of the kidney play an important role in disease maintenance and in transmission via infected urine [1].

Based on DNA-DNA hybridization and 16S rRNA phylogeny, Leptospira can be classified into more than 22 species located within three groups: the pathogenic, intermediate and non-pathogenic groups. In addition, more than 300 serovars have been described based on antigenic diversity in the lipopolysaccharide (LPS) structure on the cell surface. These different serovars can be identified using the cross-agglutination absorption test (CAAT) [3].

Pathogenic L. interrogans serovar Lai strain 56601 was the first leptospire to be subjected to genome sequencing, and the data obtained revealed the unique physiological characteristics and virulence factors of this strain [5]. Subsequently, the genomes of a number of other both pathogenic and non-pathogenic Leptospira strains have been subjected to next-generation sequencing (NGS) to investigate the genetic basis of pathogenesis [6]. Using comparative genomic analysis, pathogenic markers were defined based on gene gain and loss events, and genome rearrangements were identified [68]. An aspect that has not been explored for Leptospira is measurement of selective pressure, inferred by non-synonymous to synonymous substitution rate in genes in conserved genome regions, signaling that the genes are under positive selection: in other species these adaptations have been shown to have an important role in species diversification and adaptation in different ecological niches and hosts [9].

In pathogenic bacteria, positive selection is an evolutionary driving force arising by mutations in protein-coding genes that are associated with bacterial adaptation to changes in their environment or host species [10]. Advantageous point mutations enhance bacterial physiological fitness or virulence factor profiles appropriate to survival under various conditions [11]. Genome-wide analyses of positive selection associated with increased rates of point mutations in specific genes has been reported for several bacteria, including Bacteroides fragilis [12], Escherichia coli [13, 14], Mycobacterium tuberculosis [15], Salmonella serotypes [16] and Pasteurella multocida [17]. To date reports of positive selection in Leptospira remain scarce, and there is still a lack of genomic information for Leptospira isolates recovered from asymptomatic animals.

In the present study, we determined the serotypes and then sequenced the genomes of two $L$. interrogans isolates that were recently recovered from the urine of asymptomatic Thai dogs. We then undertook comparative analysis with these genomes and 22 other representatives of validly published Leptospira genomes obtained from the NCBI database. The Thai L. interrogans strains were subjected to genomic characterization and phylogenetic analysis to examine evolutionary relationships. Within-species, comparative genome analysis was performed with 97 strains of $L$. interrogans to obtain insight into the pan-genome structure. Strain-specific genes of the two Thai strains that were associated with specific functions including virulence also were identified. Furthermore, a genome-wide analysis for positive selection was undertaken on the core genes of L. interrogans to determine genes involved in adaptive evolution. The results provide insight into the Leptospira genome and contribute to a more comprehensive understanding of factors involved in molecular pathogenesis and adaptive evolution in pathogenic Leptospira.

\section{Results}

\section{Genome characteristics of the two Thai $L$. interrogans strains}

The basic genome characteristics of all analyzed Leptospira strains are summarized in Table 1. For L. interrogans strain CUDO5, the A5-Miseq de novo genome assembler generated 163 scaffolds with 124.47 times genome coverage and an N50 of $111,967 \mathrm{bp}$ : the genome size was estimated at $4.94 \mathrm{Mbp}$ with a $35.1 \%$ GC content. For strain CUDO8, 83 scaffolds were obtained with 109.34 times genome coverage and an N50 of 165,528 $\mathrm{bp}$, with a genome size of approximately $4.91 \mathrm{Mbp}$ and a 35.1\% GC content. PROKKA annotation showed that strains CUDO5 and CUDO8 had 4040 and 4013 predicted coding sequences (CDSs), respectively. Both strains only had one copy of each of the three ribosomal RNA genes (5S, $16 \mathrm{~S}$ and 23S). RAST categorized the functions of the CDSs in strains CUDO5 and CUDO8 into 323 and 320 sub-systems containing 1896 and 1936 CDSs, respectively. Most CDSs in strains CUDO5 and CUDO8 respectively related to RNA metabolism (288/ 1896 and 296/1936), cofactors, vitamins, prosthetic groups, pigments (284/1896 and 291/1936), amino acids and derivatives (194/1896 and 192/1936), protein metabolism (159/1896 and 167/1936), carbohydrates (149/ 1896 and 155/1936), cell wall and capsule (131/1896 and $115 / 1936)$, and motility and chemotaxis (103/1896 and 
Table 1 Representative Leptospira species and strains used in the study

\begin{tabular}{|c|c|c|c|c|c|c|c|c|c|}
\hline Species & Strain & Serovar & $\begin{array}{l}\text { Species of } \\
\text { origin }\end{array}$ & $\begin{array}{l}\text { Genome } \\
\text { status }\end{array}$ & $\begin{array}{l}\text { Size } \\
\text { (Mbp) }\end{array}$ & $\begin{array}{l}\text { Number of } \\
\text { CDS }\end{array}$ & GC\% & Accession number & $\begin{array}{l}\text { Abbreviated name in } \\
\text { this study }\end{array}$ \\
\hline L. interrogans & CUDO5 & Paidjan & Dog & Draft & 4.94 & 4040 & 35.1 & NKYH02000001.1 & CUDO5 \\
\hline L. interrogans & CUDO8 & Dadas & Dog & Draft & 4.91 & 4013 & 35.1 & NKYG00000001.1 & CUDO8 \\
\hline L. interrogans & 56601 & Lai & Human & Complete & 4.62 & 3776 & 35.0 & NC_004342.2 & LA \\
\hline L. interrogans & $\begin{array}{l}\text { Fiocruz } \\
\text { L1-130 }\end{array}$ & Copenhageni & Human & Complete & 4.62 & 3680 & 35.0 & NC_005823.1 & LIC \\
\hline L. interrogans & LP101 & Autumnalis & Human & Draft & 5.03 & 4111 & 35.2 & NZ_AHNF02000127.1 & AUT \\
\hline L. interrogans & LT1649 & Djasiman & Human & Draft & 4.67 & 3740 & 34.9 & NZ_AFMB02000229.1 & DJAS \\
\hline L. interrogans & PigK151 & Batislava & Pig & Complete & 4.72 & 3775 & 35.0 & NZ_CP011410.1 & BTL \\
\hline L. interrogans & L1111 & Bataviae & Human & Draft & 4.85 & 3984 & 35.1 & NZ_AHND02000082.1 & BTV \\
\hline L. interrogans & $\begin{array}{l}\text { Fiocruz } \\
\text { LV133 }\end{array}$ & Canicola & Human & Draft & 4.71 & 3810 & 35.0 & NZ_AKWU02000089.1 & CAN \\
\hline L. interrogans & UI 08368 & Grippotyphosa & Human & Draft & 4.87 & 3910 & 35.0 & NZ_AHNJ02000078.1 & GRP \\
\hline L. interrogans & AKRFB & Pomona & Cattle & Draft & 4.62 & 3724 & 35.0 & NZ_LUHH01000024.1 & PMN \\
\hline L. interrogans & $\mathrm{R} 168$ & Pyrogenes & Human & Draft & 4.86 & 3979 & 35.2 & NZ_AHNH02000099.1 & PYR \\
\hline L. interrogans & Norma & Hardjo & Cattle & Complete & 4.76 & 3876 & 35.0 & NZ_CP012603.1 & HJ \\
\hline L. kirschneri & $3522 \mathrm{CT}$ & Cynopteri & Bat & Draft & 4.40 & 3605 & 35.9 & NZ_AHMN02000024.1 & CYN \\
\hline L. noguchii & CZ 214 & Panama & Opossum & Draft & 4.71 & 4048 & 35.5 & NZ_AKWY02000035.1 & NOGU \\
\hline $\begin{array}{l}\text { L. } \\
\text { borgpetersenii }\end{array}$ & L550 & Hardjo-bovis & Human & Complete & 3.89 & 3606 & 40.2 & NC_008508.1 & LBL \\
\hline L. weilii & LNT1234 & undetermined & Human & Draft & 4.26 & 3876 & 40.4 & NZ_AHNC02000083.1 & WEIL \\
\hline L. alexanderi & L 60 & Manhao 3 & Human & Draft & 4.22 & 3994 & 40.2 & NZ_AHMT02000065.1 & ALEX \\
\hline L. alstonii & $80-412$ & Pingchang & Frog & Draft & 4.59 & 4139 & 42.4 & NZ_CP015217.1 & ALS \\
\hline L. santarosai & $1342 \mathrm{~K}$ & Shermani & Human & Draft & 4.03 & 3600 & 41.8 & NZ_CP006694.1 & SANTA \\
\hline L. kmetyi & Bejo-Iso9 & Malaysia & Soil & Draft & 4.41 & 3994 & 44.8 & NZ_AHMP02000004.1 & KMET \\
\hline L. licerasiae & VAR010 & Varillal & Human & Draft & 4.21 & 3865 & 41.1 & NZ_AHOO02000014.1 & LINC \\
\hline L. wolffii & Khorat-H2 & Khorat & Human & Draft & 4.40 & 4036 & 45.6 & NZ_AKWX02000023.1 & WOLF \\
\hline L. biflexa & Patoc & Patoc I (Paris) & Water & Complete & 3.95 & 3687 & 38.9 & NC_010602.1 & LBF \\
\hline
\end{tabular}

105/1936) (Fig. 1). By comparison within and between different strains, the number of CDSs in Leptospira species varied from 3600 to 4342 by PROKKA annotation. In RAST analysis $L$. santarosai and $L$. interrogans serovar Lai had the lowest and highest number of CDSs, at 1513 and 1952, respectively (Additional file 1: Table S1).

\section{Prophages, CRISPR/Cas and putative virulence factors}

According to PHASTER analysis, no intact bacteriophage (phage) was identified in strains CUDO5 or CUDO8. However, incomplete phages with sizes ranging from 6.1 to $11.4 \mathrm{Kbp}$ were found in both strains: these included PHAGE_Synech_ACG_2014b_NC_027130, PHAGE_Synech_ACG_2014f_NC_026927, PHAGE_Psychr_pOW20_A_NC_020841 and PHAGE_Sphing_PAU_NC_019521. None of these predicted phages contained any sequences associated with known pathogenicity islands or antimicrobial resistance genes. Most of the Leptospira species and strains contained incomplete phages with sizes ranging from 4.1 to $13.8 \mathrm{Kbp}$, but the only one shared was PHAGE_Sphing_PAU_NC_019521, which was found in 11 of the $13 \mathrm{~L}$. interrogans strains (Additional file 1: Table S2).

A CRISPR-Cas system was identified in strains CUDO5, CUDO8 and in some of the other strains using the CRISPRone web tool. Interestingly, non-pathogenic L. biflexa lacked this system. Direct repeats (DRs) were not identified in the genomes of $L$. borgpetersenii, $L$. alstonii, L. kmetyi, L. lincerasiae and L. wolffi; however, specific Cas proteins related to the CRISPR-Cas system were predicted in these Leptospira species. In this study, we followed the CRISPR-Cas system classification criteria that broadly categorize the CRISPR-Cas system into two classes (Class 1 and Class 2). The CRISPR-Cas system class 1 (CRISPR1) consists of three types (type I, III and IV), whilst the CRISPR-Cas system class 2 (CRISPR2) harbors two types (type II and V), with subdivision into 16 distinct subtypes based on different combinations of Cas protein [18]. Based on the presence of specific Cas proteins, several CRISPR1 subtypes were 


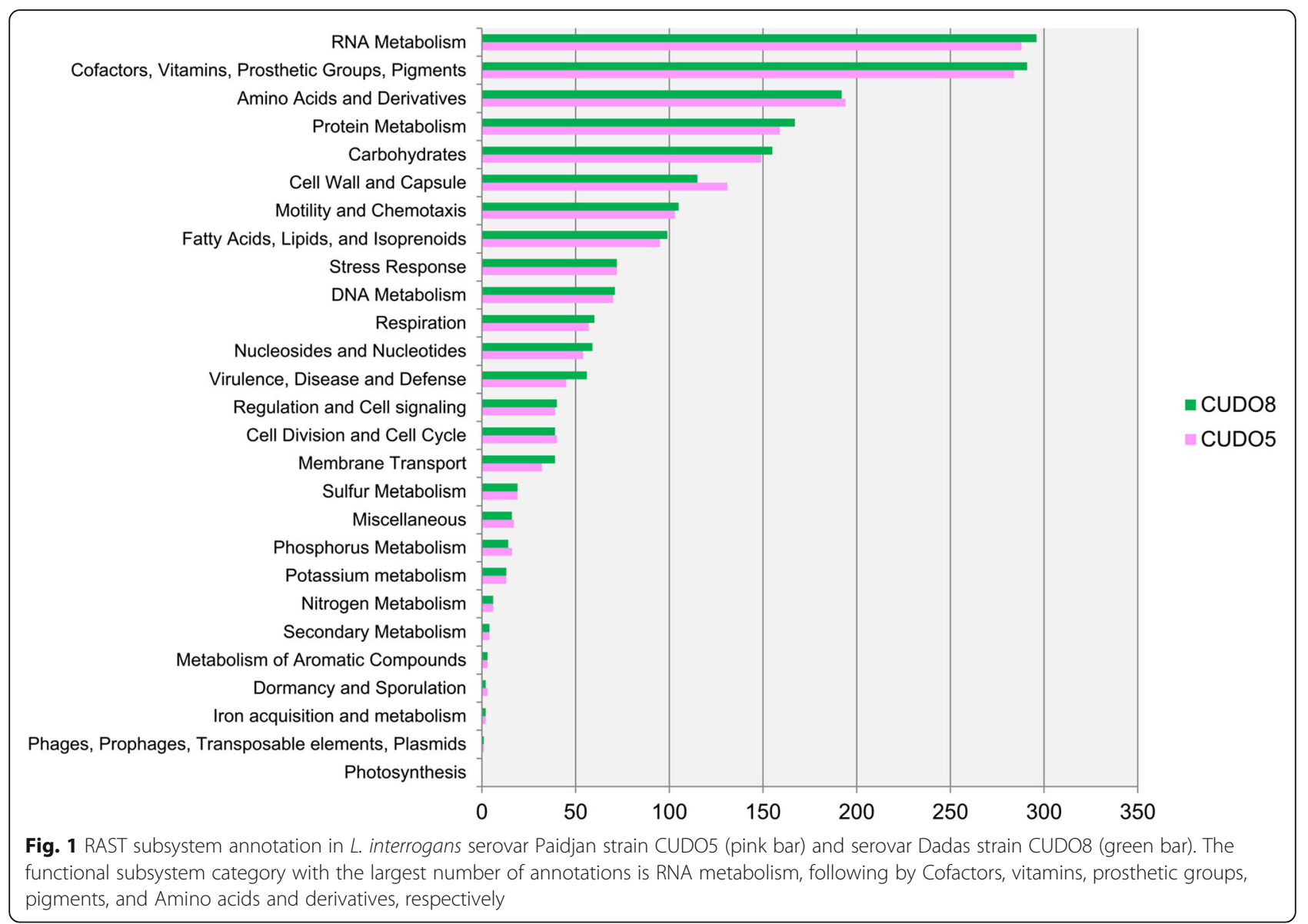

a Class 1 (subtype I-B)

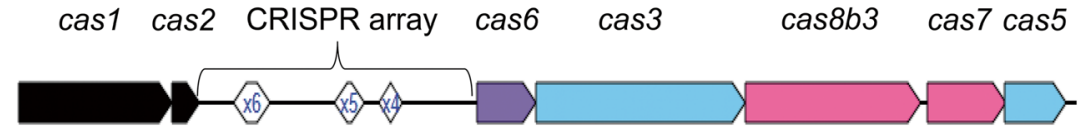

\section{Class 1 (subtype I-C)}

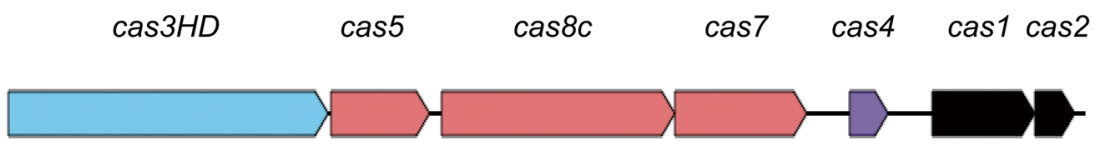

b

\begin{tabular}{lll}
\hline Species & Direct repeat consensus & Number of spacers \\
\hline L. interrogans strain CUDO5 & TTTCAATGCACTTCCTCTTGAATAAGAGAAAGAAAC & 3 \\
& CTGAATATAACTTTGATGCCGTTAGGCGTTGAGCAC & 12 \\
\multirow{2}{*}{ L. interrogans strain CUDO8 } & TTGTAAATTCTGTAGTTGTCCTATTTCCTTAGGAA & 10 \\
& AACTCACAACTCTTCCGAAAGAAATCGGACAACTACAGAATTTACAA & 11 \\
& TGCTCAACGCCTAACGGCATCAAAGTTATATTCAG & 15 \\
\hline
\end{tabular}

Fig. 2 CRISPR-Cas gene organization in L. interrogans serovar Paidjan strain CUDO5 and serovar Dadas strain CUDO8. Strains CUDO5 and CUDO8 contained CRISPR1 subtypes I-B and I-C. CRISPR1 subtype I-B is a complete locus flanked with a CRISPR array which harbors specific direct repeats (DRs) and spacers (panel $\mathbf{a}$ ), with different quantities based on the time of exogenous DNA exposure (panel $\mathbf{b}$ ) 
predicted in representative Leptospira species, including subtypes I-A, I-B, I-C and I-E (Additional file 1: Table S3). Among the L. interrogans strains, including CUDO5 and CUDO8, two CRISPR1 loci (subtype I-B and subtype I-C) were found (Fig. 2a). The CRISPR1 subtype I-B locus contained seven cas genes (cas1, cas2, cas3, cas5, cas6, cas7 and cas8b3) flanked with different numbers of DRs and spacers, while the CRISPR1 subtype I-C was an orphan locus that harbored seven cas genes (cas1, cas2, cas3HD, cas4, cas5, cas7 and cas8c) without a flanking CRISPR array region. The CRISPR array region of strain CUDO5 consisted of 4 DRs with nucleotide lengths from 36 to $39 \mathrm{bp}$ and 15 spacers, whereas strain CUDO8 harbored 4 DRs with nucleotide lengths from 35 to 48 bp and had 36 spacers (Fig. 2b). Spacer sequences predicted from CRISPRone were used to find CRISPR RNA (crRNA) targeting specific foreign sequences using the CRISPRTarget web tool. The plasmid lcp1 of $L$. interrogans serovar Linhai strain 56609 and Synechococcus phage ACG-2014b gave maximum matching scores in strain CUDO5. On the other hand, Leptospira phage LnoZ_CZ214 and plasmid lcp3 of L. interrogans serovar Linhai strain 56609 showed maximum matching scores in strain CUDO8 (Fig. 3).

By searching in the VFDB database, the genomes of $L$. interrogans strains CUDO5 and CUDO8 were shown to encode 518 and 512 putative virulence factors, respectively. The predicted virulence genes were involved with motility, chemotaxis, toxins, adhesion molecules and iron uptake systems, amongst others (Additional file 1: Tables S5 and S6). The BLAST results for gene orthologous identification among representative Leptospira strains using amino acid sequences from 33 confirmed virulence factors showed that BtuE, ClpB, FlaA, FliY, HbpA, HemO, LipL45, Loa22, Mce,
OmpL1, TlyA, TlyB and TlyC were distributed in all Leptospira strains. Genes encoding KatE, HtpG, ColA, Lig and Sph tended to be restricted to pathogenic Leptospira strains. Strains CUDO5 and CUDO8 were found to possess all core virulence-associated genes involved in acute and chronic leptospirosis infection (Fig. 4) (Additional file 1: Table S7).

\section{Serotyping, rfb locus, and lipid A and sialic acid biosynthesis encoding genes}

In the current study, serotyping using pAbs and mAbs showed that $L$. interrogans strain CUDO5 belonged to serogroup Bataviae (pAbs titer $=1: 640$ ), with close similarity to $L$. interrogans serovar Paidjan (mAbs titer = 1:20480), whilst strain CUDO8 belonged to serogroup Grippotyphosa (pAbs titer $>1: 1280$ ) with antigenic similarity to L. kirschneri serovar Dadas (mAbs titer $=$ 1:20480).

Analysis of the lipopolysacharide biosynthesis ( $r f b$ ) locus from the whole genome sequence data demonstrated that serovar Paidjan (strain CUDO5) contained nucleotide sequences of approximately $116 \mathrm{Kbp}$, which was larger than for serovar Dadas (strain CUDO8) that harbored nucleotides of around $114 \mathrm{Kbp}$. These regions encoded 125 and 113 genes for serovar Paidjan and Dadas, respectively (Additional file 1: Tables S8 and S9). In agreement with a previous report [6], the upstream and downstream flanking genes in the $r f b$ locus were conserved, with petE encoding copper-binding protein and rpsF encoding 30S ribosomal protein S6 being present in both serovars (Additional file 2: Figure S1). The complete gene cluster of dTDP-rhamnose biosynthesis $(r f b A B C D)$ and genes related to rhamnose containing glycan $(r f b B$ and $g a l E)$ were found in these two

\section{L. interrogans strain CUDO5}

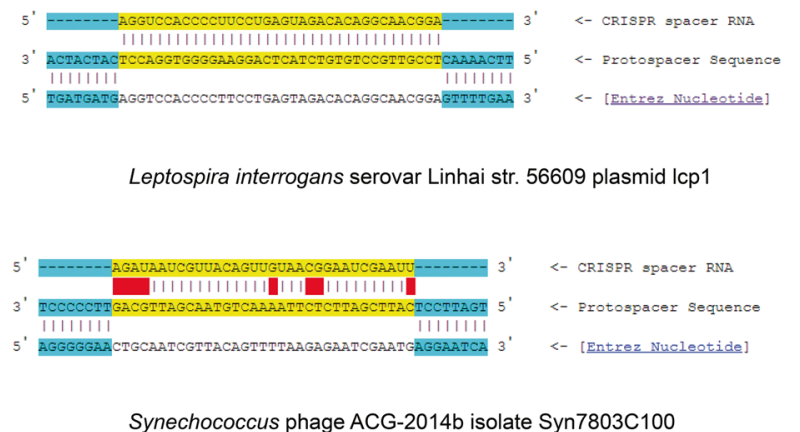

\section{L. interrogans strain CUDO8}

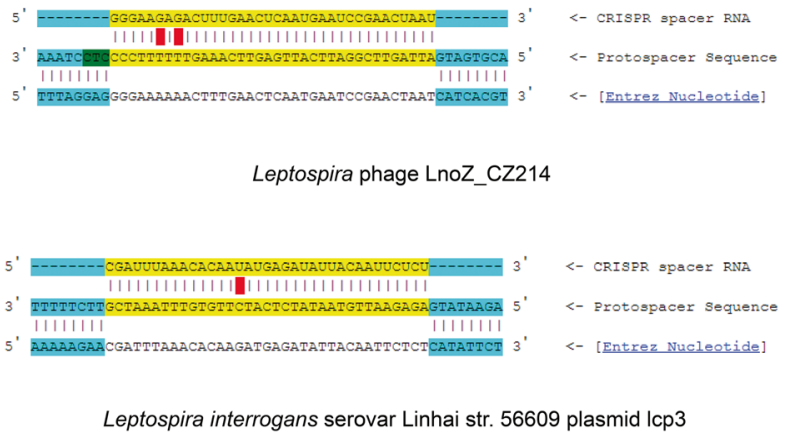

Leptospira interrogans serovar Linhai str. 56609 plasmid Icp3

Fig. 3 Graphical display of CRISPR RNA (crRNA) and target protospacer DNA in L. interrogans serovar Paidjan strain CUDO5 and serovar Dadas strain CUDO8. Spacer specific sequences are transcribed to crRNA to protect against phage and plasmid re-infection. The crRNA in strains CUDO5 and CUDO8 gave highest matching scores to a plasmid of L. interrogans serovar Linhai strain 56609. Colored yellow indicates the complementary matching between spacer and target protospacer sequence; red indicates the mismatching of spacer and target protospacer sequence and blue represents the flanking sequence between spacer and target protospacer 


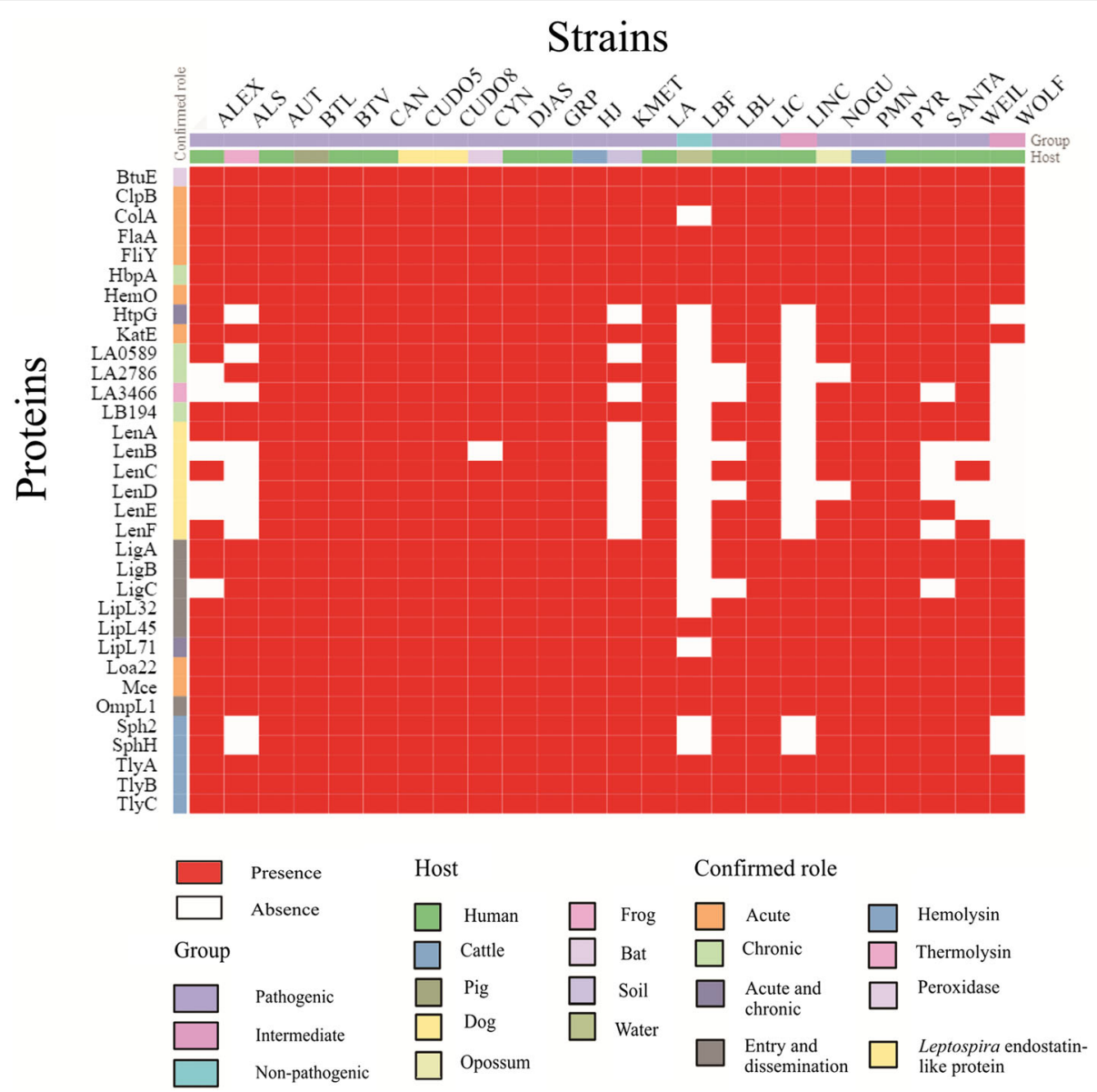

Fig. 4 The distribution and conservation of 33 representative confirmed virulence genes in pathogenic, intermediate and non-pathogenic Leptospira species. Genes encoding for outer membrane protein (loa22), flagella motor switch protein (fliY), and hemolysins B and C (tlyB and tlyC) were conserved in all 24 Leptospira strains. Gene involved with chronic infection (Ib194) was conserved only in pathogenic Leptospira strains. Strains CUDO5 and CUDO8 contained all of the 33 virulence genes

rfb loci. Besides rhamnose biosynthesis genes, serovar Dadas contained additional genes involved with capsular heptose biosynthesis ( $f c l, g m h A$ and $r f b C)$. Sialic acid biosynthesis genes including nеuA1 and neuB2 were found within the $r f b$ locus of serovar Paidjan. The gene encoding bacillosamine/legionaminic acid aminotransferase $(p g l E)$, which plays a role in protein glycosylation via the $N, N^{\prime}$-diacetylbacillosamine biosynthesis pathway, was found in both serovars. Three aminotransferases belonging to the DegT family that are involved with LPS $\mathrm{O}$-antigen side chain biosynthesis were identified in serovar Paidjan. Genes related to the Wzx/Wzy-dependent pathway ( $w z x$ and $w z y$ ) were not situated in the $r f b$ locus of either strain, but were located elsewhere in the genome (BLASTP analysis). A total of 11 enzymes for lipid A biosynthesis encoded by $h t r B, k d s A, k d s B 2, k d t A$, lnt, $l p x A, l p x B 1, \operatorname{lp} x C, l p x D 1, l p x D 2$ and $l p x K$ were present across the Thai Paidjan and Dadas genomes, but both lacked $l p x B 2$ and $k d s B 1$ (BLASTP analysis) that are found in L. interrogans serovar Lai strain 56601 [6].

\section{Average nucleotide identity (ANI) and phylogenomic analysis}

The results from the JSpecies analysis showed that the ANI percentages among the genus Leptospira varied widely from 64.17 to $99.19 \%$ for pairwise genome comparisons in the pathogenic, intermediate, and non-pathogenic Leptospira groups. For example, genomic comparison between $L$. wofflii and L. biflexa revealed $64.17 \%$ nucleotide similarity, whereas $L$. interrogans serovar Copenhageni and $L$. interrogans serovar Djasiman had $99.19 \%$ nucleotide similarity (Additional file 3: Table S1). Moreover, in 13 representative $L$. interrogans strains, the ANI values gave a similarity percentage of more than $98 \%$. Strains CUDO5 and CUDO8 had the highest ANI values to each other (99.08\%).

For phylogenomic analysis of L. interrogans strains, a total of 87,442 concatenated core-SNPs from the 97 genomes (Additional file 3: Table S2) were used to generate a maximum likelihood phylogenetic tree with the GTR nucleotide substitution model. All strains were obtained 
from different geographical regions and hosts. The phylogenetic tree revealed two defining features: serovar specific branches, and a connectivity of human and animal isolates. Some serovars were clustered in the same clades, with bootstrap percentage of more than 75 supported, including serovar Australis (strains 200,703,203, SU5 and LT2148), serovar Grippotyphosa (strains Andaman, 2006006986, UI08368 and LT2186), serovar Hardjo (strains Prajitno, OV5 and Norma), serovar Pomona (strains UT364, GR5, AKRFB, Pomona and CSL 4002), and serovar Pyrogenes (strains 200701872, R168, L0374 and Sri Lanka 30). These strains came from different geographical locations. Notably, an intermingling of human and animal isolates was identified, particularly in the cluster of serovar Australis (human and pig), Hardjo (human and cattle), Pomona (human, pig and cattle) and Pyrogenes (human and rodent). L. interrogans serovar Paidjan strain CUDO5 and serovar Dadas strain CUDO8 were grouped in the same branch, with a bootstrap percentage of 100. Moreover, strains CUDO5 (serovar Paidjan) and CUDO8 (serovar Dadas) isolated from the urine of asymptomatic dogs were clustered on the evolutionary tree with leptospiral isolates recovered from humans in Thailand and China (Fig. 5).

\section{Pan-genome and core-genome analysis, and identification} of strain-specific genes in the two Thai canine strains

Using the power fit law equation, it was calculated that $b$ $=0.167923$, and the expected size of the L. interrogans pan-genome was 7782 gene families. The pan-genomes tended to increase after each new genome addition (Fig. 6a), and were assumed to be open in this analysis ( $n$ =97). In contrast, the core-genome gradually decreased and became constant after 80 genomes were analyzed (Fig. 6a).

The core gene set across the $L$. interrogans strains $(n=$ 97) was made up of 734 gene families, whilst the accessory gene set varied from 2062 to 3303 gene families (Additional file 3: Table S3). The functional classification of the gene repertoire in core- and strain-specific genes according to COG categories was different. Core-genes encoding for proteins related to category J (Translation, ribosomal structure and biosynthesis), E (Amino acid transport and metabolism) and R (General prediction only) were abundant among the total core-genes identified, whereas strain-specific genes encoding for proteins involved in categories R (General function prediction only), L (Replication, recombination and repair) and $\mathrm{M}$ (Cell wall/membrane/envelope biogenesis) were over-assigned among this set of genes. The COG distribution of the core-, accessory and strain-specific genes are shown in Fig. 6b.

The pan-genome analysis of the $97 \mathrm{~L}$ interrogans strains identified genes that were only found in the two Thai canine strains (i.e. were specific to them). $L$. interrogans strain CUDO5 contained eight strain-specific genes, of which seven could not be classified by eggNOG; hypothetical protein. One gene was found using an NCBI database search, and its predicted product resembled a type III restriction endonuclease. In strain CUDO8, BPGA identified three strain-specific genes, of which two were classified as hypothetical proteins, and the other was assigned as phage terminase large subunit.

\section{Positive selection in the $L$. interrogans genome and mapping on protein tertiary structure}

According to GET_HOMOLOGUES, a total of 2721 single-copy orthologous genes were shared among the 13 representative $L$. interrogans strains, and these were defined as core genes. After eliminating incomplete sequences due to frameshift mutations, insertions and deletions, 2408 protein-coding sequences were left. Of these, $56.36 \%$ of the encoded proteins had known function and the rest were hypothetical proteins. This study focused only on proteins of known function. Based on the LRT statistic for the site-model, 352 genes were under significant positive selection (FDR $<5 \%$ ). Genes under positive selection were further identified based on functional classification and on whether they encoded known virulence factors, using the COG and VFDB categories respectively. In relation to functional classification, COG classified protein-coding genes involved with category $M$ (cell wall/envelope biosynthesis), E (amino acid transport), $\mathrm{H}$ (coenzyme transport and metabolism), $\mathrm{T}$ (signal transduction mechanism) and $\mathrm{J}$ (Translation, ribosomal structure and biogenesis) were significantly over-represented among the genes showing evidence of positive selection $(P<0.05$, one-side binomial test) (Additional file 4: Table S1). Of these genes under positive selection, 101 were predicted as encoding putative virulence factors. Most of the genes in the three groups adhesion, anti-phagocytosis and iron uptake system were significantly over-represented $(P<0.05$, one-side binomial test) (Additional file 4: Table S2). Moreover, another 74 genes showed evidence for positive selection and recombination (Additional file 4: Tables S3 and S4, Additional file 5: Figure S1). Interestingly, colA encoding for collagenase was identified amongst these, and this had an amino acid sequence homology of $29.19 \%$ to the enzyme in Clostridium perfringens. Positional mapping of 101 predicted virulence genes under positive selection is shown in Fig. 7, and a summary of statistical analyses relating to positive selection for 18 representative virulence genes related to adhesion, anti-phagocytosis, iron uptake system, LPS biosynthesis, exotoxin and motility of L. interrogans is presented in Table 2.

To obtain more insight into positive selection based on protein tertiary structure, amino acid residues under positive selection were mapped on $3 \mathrm{D}$-modeled protein 


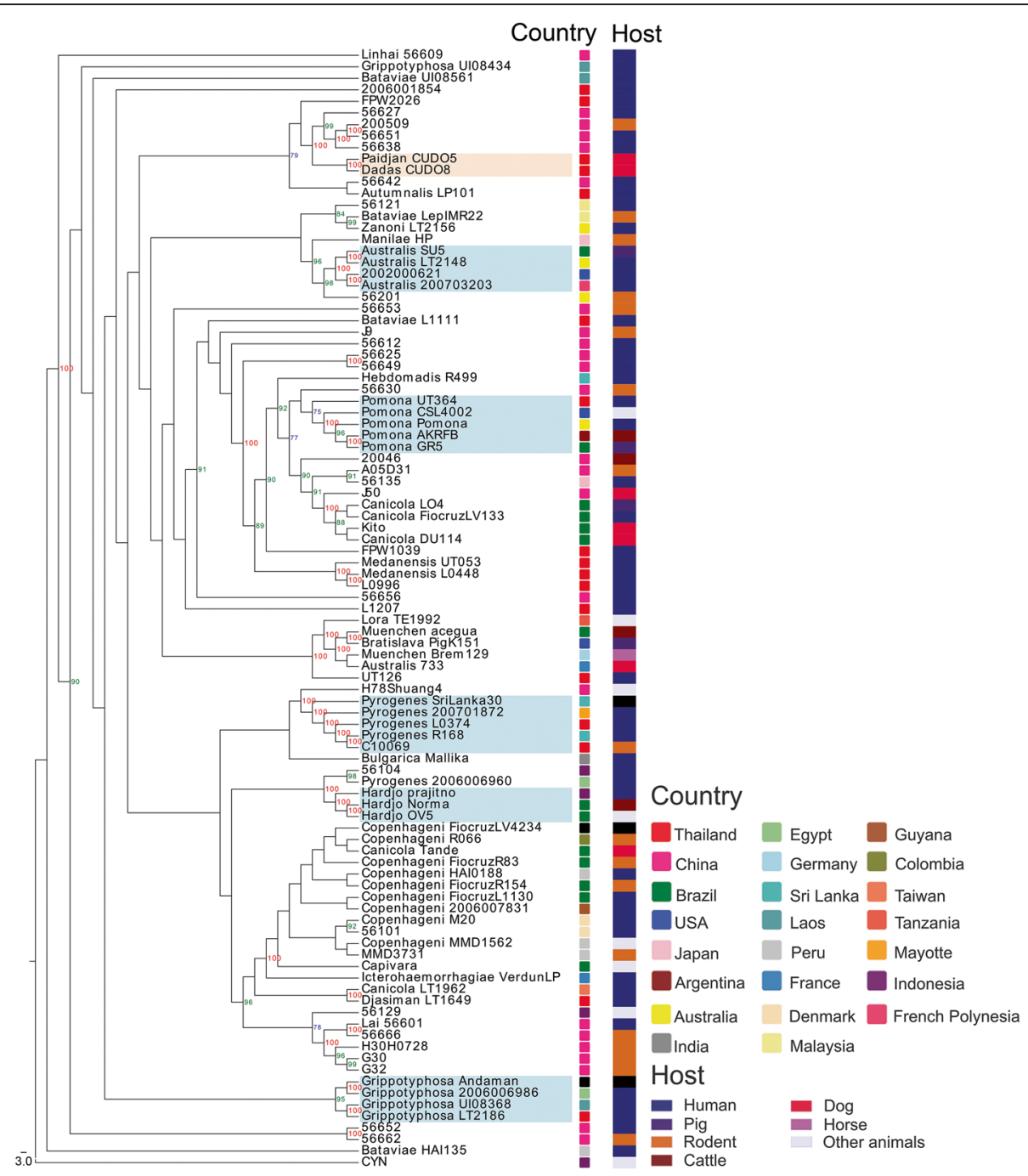

Fig. 5 Whole genome core-single nucleotide polymorphisms (core-SNPs) phylogeny of L. interrogans based on the maximum likelihood method with 1000 bootstrap replicates. The core-SNPs phylogenomic analyses was visualized by EvolView [63]. The tree shows that L. interrogans serovar Paidjan strain CUDO5 and serovar Dadas strain CUDO8 were placed in the same branch with a bootstrap percentage of 100 as highlight in peach color. Serovar specific clades with bootstrap percentage of more than of 75 are highlight in blue color, including serovar Australis, Grippotyphosa, Hardjo, Pomona and Pyrogenes. L. kirscheneri serovar Cynopteri strain 3522 CT (CYN) was used as an outgroup in this study

structures. Of the 101 genes under positive selection and predicted to be related to putative virulence in Leptospira, four representative proteins that have been experimentally confirmed to be virulence factor of pathogenic Leptospira were selected for this analysis: hemolysin A (encoded by tlyA); TonB-hemin binding receptor (encoded by $h b p A$ ); flagella motor switch protein (encoded by fliN2); and UDP-3-O-(3-hydroxymyristoryl) glucosamine $N$-acyltransferase (encoded by $l p x D 1$ ). These all had values of $\omega>1$, significant $q$-values, and a high confidence of obtaining 3D-protein-based homology predictions. The tlyA, hbpA, fliN2 and $l p x D 1$ genes encoded proteins made up of 259, 776, 178 and 340 amino acid residues, respectively.
The results from the BEB analysis revealed that three amino acids in L. interrogans TlyA (amino acid positions 40, 43 and 258), two in HbpA (positions 240 and 395), 11 in FliN2 (positions 54, 58, 63, 65, 67, 68, 69, 70, 72, 74 and 75) and one in LpxD1 (position 47) were subjected to positive selection with a high posterior probability $(\mathrm{PP}>0.95)$. According to the protein tertiary mapping by PyMOL, positive selection of TlyA occurred in the loop connecting two $\alpha$-helices and the end of the C-terminus (Fig. 8a), whereas for HbpA, positive selection was present in the loop connecting two $\beta$-pleated sheets (Fig. 8b). In FliN2, positive selection occurred in the loop linking two $\alpha$-helices (Fig. 8c), while for LpxD1 positive selection was detected within a $\beta$-plated strand 

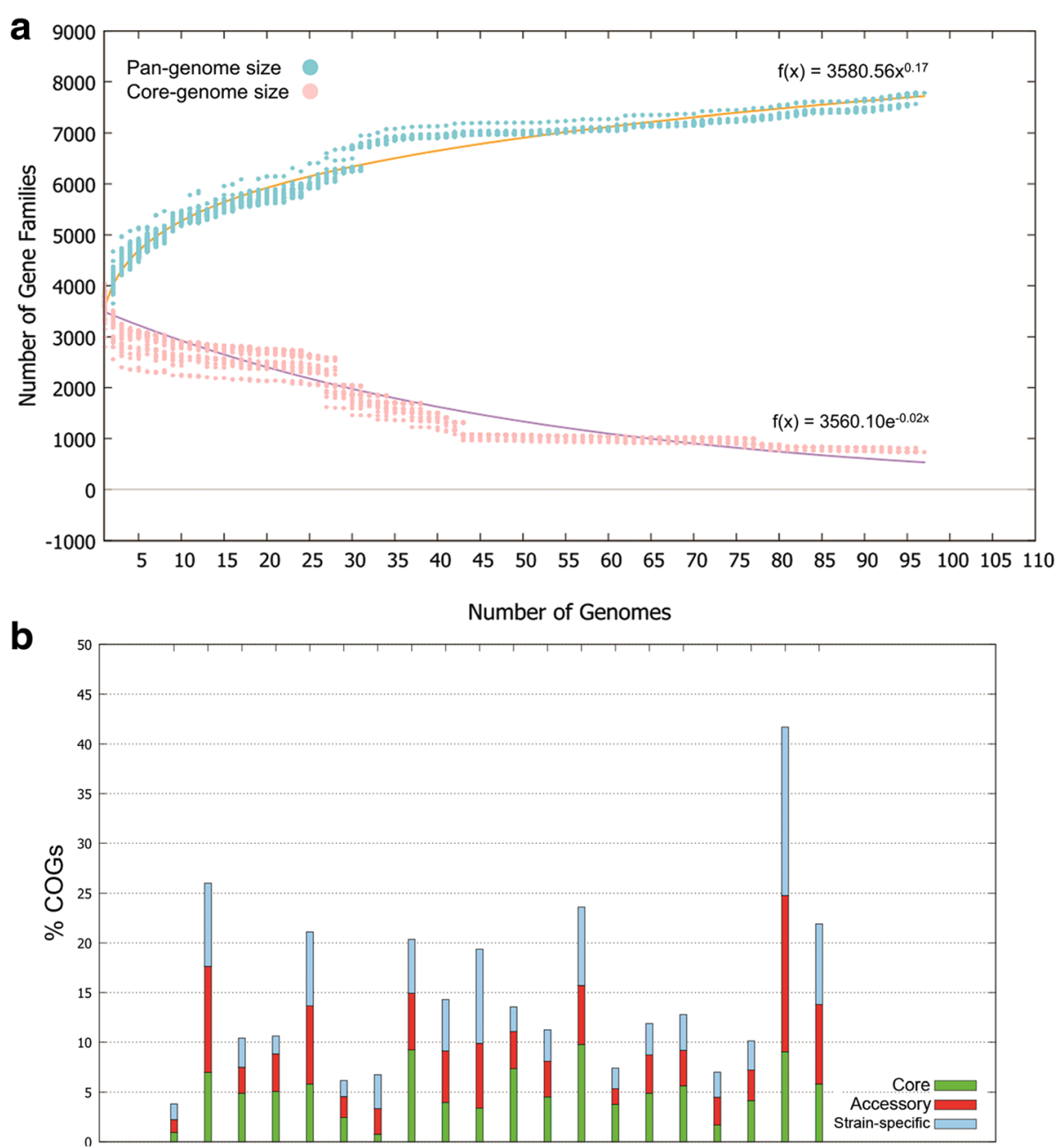

DM N O T U V J K L C G F H I Q P R S

Fig. 6 Pan-genome analysis of L. interrogans $(n=97)$ using the BPGA pipeline. The power and exponential law equations were used to create pan- and core-genome curves of L. interrogans (panel a). The COG categories distribution of core, accessory and strain-specific genes in $97 \mathrm{~L}$. interrogans strains are shown in panel $\mathbf{b}$, and according to Heap's law the Leptospira genome was predicted to be open. The COG categories are denoted as follows: D (Cell cycle control, cell division, chromosome partitioning), M (Cell wall/membrane/envelope biogenesis), N (Cell motility), O (Posttranslational modification, protein turnover, chaperones), T (Signal transduction mechanisms), U (Intracellular trafficking, secretion, and vesicular transport), V (Defense mechanisms), J (Translation, ribosomal structure and biogenesis), K (Transcription), L (Replication, recombination and repair), C (Energy production and conversion), G (Carbohydrate transport and metabolism), E (Amino acid transport and metabolism), F (Nucleotide transport and metabolism), H (Coenzyme transport and metabolism), I (Lipid transport and metabolism), Q (Secondary metabolites biosynthesis, transport and catabolism), P (Inorganic ion transport and metabolism), R (General function prediction only), and S

(Function unknown)

(Fig. 8d). Moreover, at more than 90\% confidence, Phyre2 modeled L. interrogans TlyA as sharing 37\% amino acid identity with a putative hemolysin of Streptococcus thermophiles; FliN2 had 44\% amino acid identity to a flagella motor switch protein homolog in Salmonella Typhimurium; HbpA shared $14 \%$ amino acid identity with a ferripyoverdine receptor in Pseudomonas aeruginosa; and LpxD1 shared 26\% amino acid identity with UDP-3-O-(3-hydroxymyristoryl) glucosamine $N$-acyltransferase in Pseudomonas aeruginosa. The Phyre2 investigator tool showed that none of the amino acid residues under positive selection in TlyA, HbpA, FliN2 or LpxD1 occurred in the pocket sites.

\section{Discussion}

The present study reports the first genomic characterization of $L$. interrogans strains isolated from dogs in Thailand, and presents a comparative genome analysis of these with the published genomes of another 12 Leptospira species. Moreover, a genome-wide scan of genes under positive selection, focusing on potential virulence factors of L. interrogans, was undertaken to obtain 


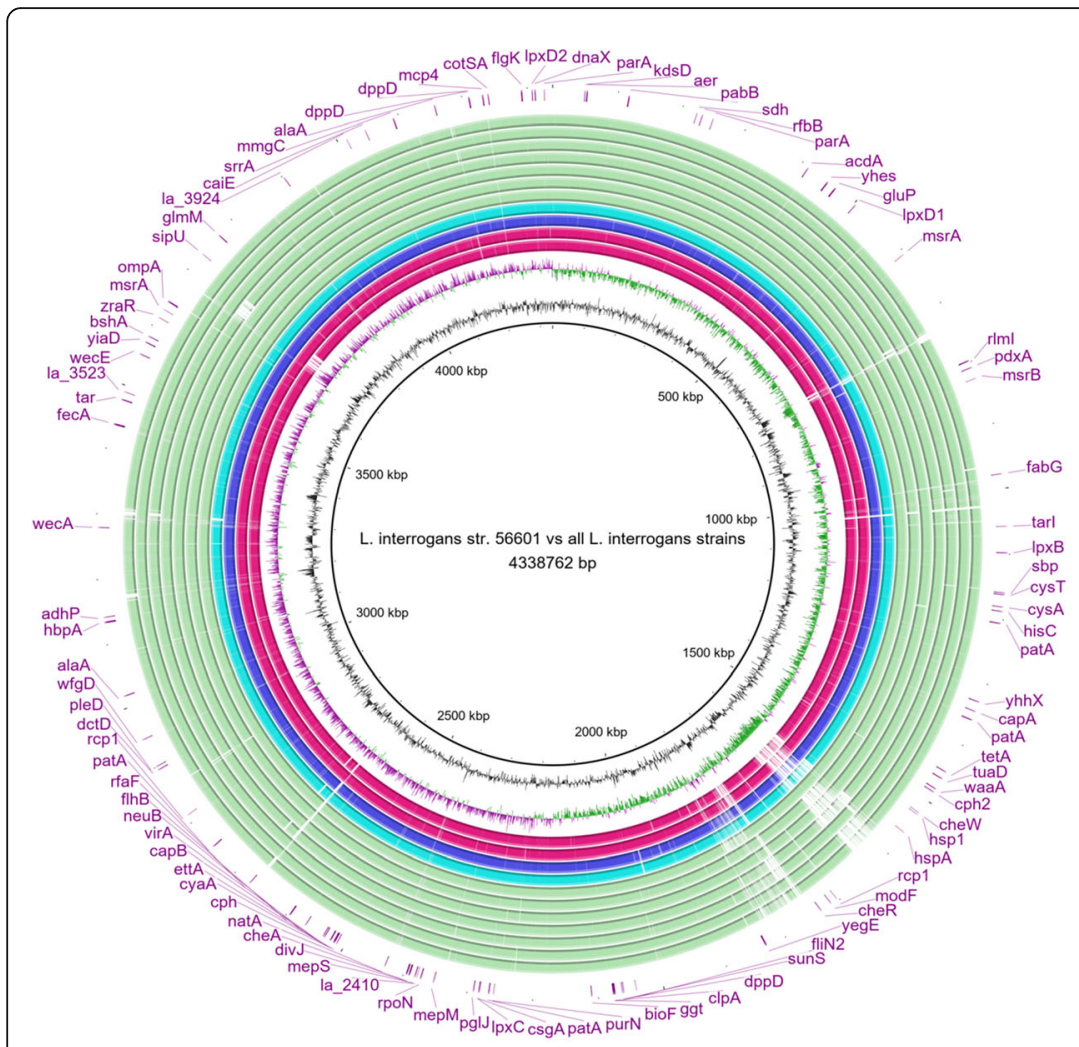

\section{Chromosome I}

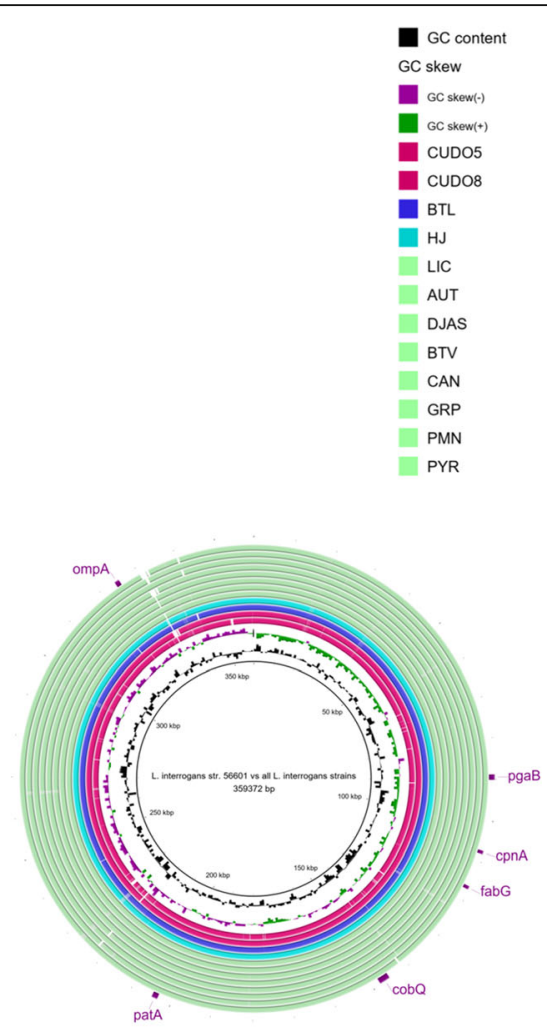

Chromosome II

Fig. 7 Circular genome map of L. interrogans serovar Lai strain 56601 and the other 13 representative strains studied, with the location of 101 predicted virulence genes under positive selection marked. Comparative sequence analysis was performed with BRIG [76] using L. interrogans strain 56601 as a reference (thick black innermost ring) against other representative related $L$. interrogans strains. The second inner ring indicates GC contents (black color) followed by the GC skew (purple and green colors). Each colored ring shows the host origin in each strain of chromosomal DNA of Leptospira presented as follows: magenta for dogs, blue for pigs, cyan for cattle and light-green for humans. The outermost labels show the location of 101 predicted virulence genes under positive selection in L. interrogans. The intensity of the ring color represents the percentage of nucleotide identity from 50 to 90

insights into genetic diversity, molecular pathogenesis and molecular adaptation of the Leptospira strains.

L. interrogans serovar Paidjan strain CUDO5 and $L$. interrogans serovar Dadas strain CUDO8 were isolated from the urine of asymptomatic dogs in an area of Thailand with a high frequency of human leptospirosis, and a $9.92 \%$ prevalence in animals. Evidence from previous rrs phylogenetic analysis has revealed close genetic links between isolates from animals and humans in this area [19]. Therefore, theses strains have epidemiological importance and potentially may pose a risk to public health in the region. The availability of genome sequences from both Thai Leptospira serovars provides useful new information relating to disease diagnosis and prevention.

Analysis of the genomes of the two Thai strains demonstrated that they had similar numbers of CDSs and sub-system categories to other analyzed $L$. interrogans strains. Most of the genes encoded proteins responsible for basic biological processes such as transcription, translation, cell wall synthesis and motility, and the findings confirm the high conservation of CDSs among $L$. interrogans strains.

Phages are viruses that infect specific bacterial hosts, and are abundant in the environment [20]. Prophage-related genes were found in the Leptospira genomes, presenting as incomplete phages without any obvious association with pathogenicity islands or antimicrobial resistant genes, but with diverse prophage types (Additional file 1: Table S3). In this study, Sphingomonas phage (PHAGE_Sphing_PAU_NC_019521) was found in 11 of the 13 representative L. interrogans strains, and this phage potentially could be exchanged between L. interrogans and Sphingonomas paucimobilis in shared environments such as in water sources [21].

The CRISPR-Cas system is defined as part of the bacterial adaptive immune system. This system can remember and destroy phages and plasmids invading the 
Table 2 Statistical summary of 18 virulence-associated genes under positive selection in 13 representative L. interorgan strains

\begin{tabular}{|c|c|c|c|c|c|}
\hline Gene & COG categories $^{a}$ & Gene annotation & $\omega^{\mathrm{b}}$ & $2 \Delta \ell^{c}$ & q-value \\
\hline \multicolumn{6}{|l|}{ Adhesion } \\
\hline$k d s D$ & M & Arabinose 5-phosphate isomerase & 15.32026 & 10.347320 & 0.011652800 \\
\hline ompA & M & Outer membrane protein $\mathrm{A}$ & 17.12571 & 20.820944 & 0.000130800 \\
\hline yiaD & M & Outer membrane protein $\mathrm{A}$ & 18.64214 & 23.296514 & 0.000046900 \\
\hline rfaF & M & Glycosyl transferase, family 9 & 20.83838 & 13.943466 & 0.002602300 \\
\hline la_2410 & M & Glycerol-3-phosphate cytidylyltransferase & 20.63787 & 11.376946 & 0.007541200 \\
\hline suns & M & Glycosyl transferase, family 2 & 77.84603 & 23.733642 & 0.000039100 \\
\hline patA & M & Membrane bound O-acyltransferase (MBOAT) family protein & 33.76757 & 34.295972 & 0.000000426 \\
\hline \multicolumn{6}{|c|}{ Anti-phagocytosis } \\
\hline rmd & M & NAD-dependent epimerase dehydratase & 15.67082 & 13.845838 & 0.002710400 \\
\hline$c a p B$ & M & Capsule biosynthesis protein & 10.13343 & 16.584998 & 0.000831400 \\
\hline capA & M & Capsule biosynthesis protein & 26.72717 & 7.6514580 & 0.037726700 \\
\hline \multicolumn{6}{|c|}{ Iron uptake system } \\
\hline fecA & M & TonB-dependent receptor plug domain & 14.34022 & 31.619632 & 0.000001330 \\
\hline hbpA & P & TonB-dependent receptor & 8.341820 & 10.766942 & 0.009678900 \\
\hline $\bmod F$ & $P$ & $A B C$ transporter & 10.62873 & 8.1123800 & 0.030958000 \\
\hline \multicolumn{6}{|c|}{ LPS biosynthesis } \\
\hline IpxD1 & M & UDP-3-O-(3-hydroxymyristoryl) glucosamine $\mathrm{N}$-acyltransferase & 65.82909 & 9.594336 & 0.016131033 \\
\hline \multicolumn{6}{|l|}{ Exotoxin } \\
\hline tly $A^{\mathrm{d}}$ & & Hemolysin A & 61.51328 & 30.989188 & 0.000001670 \\
\hline \multicolumn{6}{|l|}{ Motility } \\
\hline flin2 & $\mathrm{N}$ & Flagella motor switch protein & 31.01218 & 35.57534 & 0.000000249 \\
\hline flgK & N & Flagella hook-associated protein & 16.72326 & 15.544766 & 0.001312461 \\
\hline$f / h B$ & $\mathrm{~N}$ & Polar flagella protein & $999.0000^{\mathrm{e}}$ & 22.055182 & 0.000076800 \\
\hline
\end{tabular}

aThe abbreviations for COG categories are defined as: M (Cell wall/membrane/envelope biogenesis), P (Inorganic ion transport and metabolism), J (Translation, ribosomal structure and biogenesis) and $\mathrm{N}$ (Cell motility)

${ }^{\mathrm{b}} \omega$ is the ratio of $d \mathrm{~N} / d \mathrm{~S}$ under positive selection (model M2a)

$\mathrm{C}_{2} \Delta \ell$ is likelihood ratio test (LRT) statistic

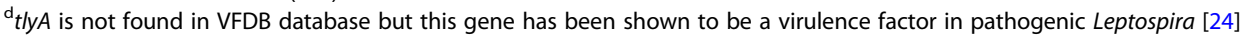

${ }^{e}$ All substitutions of $f l h B$ are non-synonymous and $d S=0$; therefore the $\omega$ value is undefined and represented as 999 in the table. According to CODEML

developer, LRT is not affected by $d S$ in this analysis as shown by a significant $q$-value result

host cell by producing specific crRNA against these elements [18]. Our results are in agreement with a previous study where the CRISPR-Cas system was found only in intermediate and pathogenic group strains, which harbored at least one subtype of CRISPR1, whereas non-pathogenic Leptospira species did not have this system [6]. The CRISPR-Cas system has been thought to be a necessary functional system in pathogenic Leptospira [6]. The strains from dogs in this study possessed two CRISPR1 loci (subtypes I-B and I-C). CRISPR1 subtype I-B targets foreign DNA invasion, while the function of subtype I-C is still uncharacterized. These two loci of the CRISPR-Cas system also are found in Pyrococcus furiosus, suggesting its ability to use crRNA to defend against a wide range of foreign genetic material, including both DNA and RNA [22]. Various crRNAs targeting either plasmids or phages in various species were identified in L. interrogans serovar Dadas strain CUDO8 $(n=40)$ and to a lesser extent in L. interrogans serovar Paidjan strain CUDO5 $(n=31)$ (Additional file 1: Table S4). The difference suggests that CUDO8 may have been subjected to infection with more phages and plasmids than CUDO5. The fact that the plasmid of $L$. interrogans serovar Linhai strain 56609 was found in CUDO5 and CUDO8 might reflect their shared evolutionary history, or transfer of the plasmid during their occupation of shared microenvironmental niches before their dissemination to different hosts. On the other hand, it should be noted that crRNAs targets predicted by bioinformatics programs quite commonly have non-specific or coincidental hits (both plasmid and phage), especially when querying with short sequences. Therefore, a CRISPR interference assay is required to validate the crRNAs target results relating to interference with foreign genetic material in Leptospira [23]. 


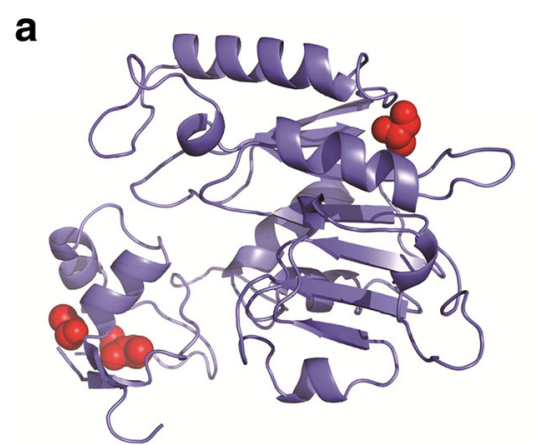

TlyA

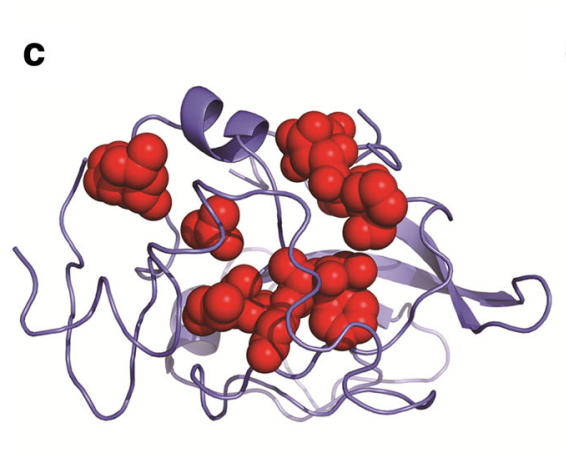

FliN2 b

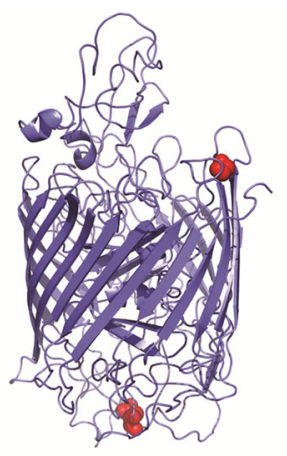

$\mathrm{HbpA}$

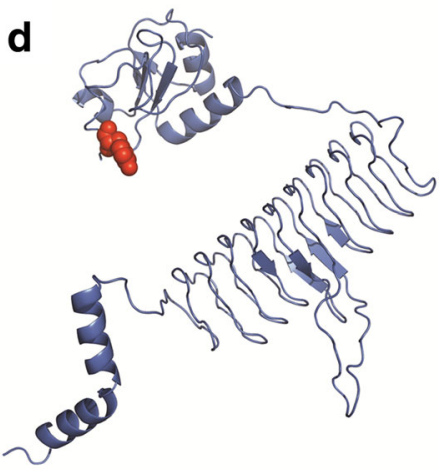

LpxD1

Fig. 8 Three-dimensional protein models of Hemolysin A (encoded by tlyA: panel a), Ton-B hemin binding receptor (encoded by hbpA: panel b), Flagella motor switch protein (encoded by fliN2: panel c), and UDP-3-O-(3-hydroxymyristoryl) glucosamine N-acyltransferase (encoded by IpxD1: panel $\mathbf{d}$ ) under positive selection in L. interrogans. Sites of strong positive selection are highlighted as red spheres (posterior probability $>0.95$ )

The presence of virulence genes associated with the pathogenesis of leptospirosis have been used to divide Leptospira species and strains into three groups: pathogenic, intermediate and non-pathogenic [24]. In this study, we identified virulence factors present in strains CUDO5 and CUDO8 genomes using the VFDB database. However, VFDB searching has some limitations since virulence genes/proteins with confirmed roles in other bacteria might not reflect their function in strains CUDO5 and CUDO8. Therefore, a combination search with orthologous virulence genes with established roles in acute and chronic leptospirosis also was undertaken. All pathogenic Leptospira strains contained genes encoding catalase, collagenase, TonB-hemin binding receptor, immunoglobulin-like protein B and sphingomyelinases, which all are confirmed as virulence markers during the early stage of infection [24]. On the other hand, intermediate and non-pathogenic Leptospira lack many of these virulence genes: their acquisition may reflect an evolutionary process favoring host infection and disease [6], whilst loss of these genes may result in attenuation and reduction in virulence. Although strains CUDO5 and CUDO8 have genes with confirmed roles as virulence factors, they were isolated from the urine of asymptomatic dogs. Their ability to establish persistent infections with long-term kidney colonization is likely to reflect a balance of gene content and expression that favors an advantageous carrier state - maximizing the opportunity for further transmission via urine.

Comparative genome analysis can be used to understand genomic diversity and provide insights into biological functions and evolution [25]. Using the power law exponents of Heap's law, our data indicated that $L$. interrogans strains have an open pan-genome [6,7]. This finding suggests that the gene pool of the spirochetes can expand, and the observed genomic plasticity probably results from multiple ways of incorporating new genetic material into their genomes such as through horizontal gene transfer and/or replication of advantageous genes via gene duplication [7]. This phenomenon is beneficial in relation to adaptations resulting in increased infectivity and survival in a broad range of mammalian hosts and the environment [1].

The Leptospira species that were analyzed were separated into three groups using DNA-DNA hybridization as well as by virulence gene profiles [6]. The 13 representative strains of $L$. interrogans had ANI values of over $98 \%$, suggesting that nucleotide sequences of $L$. interrogans strains are mostly conserved, even though the 
strains were of different serovars and were from different origins and epidemiological units. The ML-based core-SNPs phylogenetic analysis identified both distinct and clustered Leptospira serovars in the same branch of the phylogenetic tree. This finding reflected the diversity of the Leptospira strains, even amongst those coming from the same geographical origins. It also inferred different accumulations of SNPs in the core genome in each strain. Moreover, there were instances of connectivity between human and animal Leptospira isolates, providing genetic evidence supporting the occurrence of disease maintenance in animals and cross-species transmission. Notably, the phylogenetic tree confirmed that the two Thai canine strains were closely related to each other. However, the serotyping results revealed that they belonged to different serogroups and serovars (serogroup Bataviae serovar Paidjan for strain CUDO5 and serogroup Grippotyphosa serovar Dadas for strain CUDO8). Consistent with the $r f b$ locus analysis, serovars Paidjan and Dadas had gene variations in these regions. Nevertheless, genes association with rhamnose biosynthesis $(r f b A B C D)$ and rhamnose containing glycan $(r f b B$ and $g a l E$ ) were distributed in both serovars. Previously, the LPS of pathogenic Leptospira has been shown to contain more rhamnose than intermediate Leptospira, and moreover specific sugars were found related to particular serovars [26]. This finding was in agreement with our results in the case of serovar Dadas that contained three additional genes associated with capsular heptose biosynthesis. Furthermore, the gene encoding aminotransferase PglE that was found in both serovars might play a crucial role in creating novel sugar composition during the glycosylation process of outer membrane proteins [27]. The three aminotransferases belonging to the DegT family in serovar Paidjan might be associated with LPS O-antigen side chain biosynthesis, leading to differences between the serological characteristics of the serovars [28]. These findings provided possible explanations for the difference in LPS composition and outer membrane protein post-translational modifications between serovars Paidjan and Dadas. However, more experiments are required to compare the relationship of the genes in the $r f b$ locus, sugar composition, sugar ramification and structural determination of the LPS, since there are large variations and complexities in this material [1]. A previous investigation of the LPS of pathogenic and intermediately pathogenic Leptospira species using multiple methods including LPS extraction, gas chromatography-mass spectrometry (GC-MS), and electron microscopy revealed significant differences in terms of carbohydrate and fatty acid composition, including in Leptospira isolated from common reservoir hosts [26]. The presence of large quantities of a specific sugar in a particular serovar is believed to play an important role for survival and colonization in certain environments [26]. Therefore, a combination of genomic detail and biochemical and structural analysis will allow a better understanding of LPS biology in the adaptation strategies in these two pathogenic Leptospira.

In bacteria, strain-specific genes are associated with specific functions and niche adaptations [25]. Strains CUDO5 and CUDO8 harbored strain-specific genes compared to the other strains in this study $(n=97)$, but these were mostly predicted as hypothetical proteins with unknown functions. Nevertheless, strain-specific genes of known function were found in these strains, consisting of; a type III restriction endonuclease and phage terminase large subunit. The function of these genes may be related to the pathogenesis of Leptospira. Notably, type III restriction endonucleases are functionally associated with defense mechanisms against invading foreign DNA. Therefore, this gene possibly functions to protect $L$. interrogans from phage infection, and could modulate the genetic exchange barrier [29]. As no complete phage was reported in this study, the cryptic prophage including phage terminase large subunit might indicate the existence of a prior phage infection that was probably degraded by the CRISPR-Cas system and/or a restriction modification system, and was subsequently trapped in the Leptospira chromosome [30]. Although the role of phage terminase large subunit in the Leptospira strains was not established, cryptic prophages in $E$. coli contribute to survival in adverse environment conditions [31]. Therefore, this cryptic prophage might help Leptospira to cope with stress in new hosts and environments.

Beneficial mutations in bacterial genes under positive selection can assist in molding bacterial-host adaptation [11], whilst recombination helps positive selection to become fixed in the population [32]. Our investigation of positive selection among the genomes of 13 representative L. interrogans strains focused on genes encoding virulence-associated proteins. Genes under significant positive selection included 101 predicted virulence-associated genes and 352 genes with various functions, including those involved in cell wall/envelope biosynthesis; amino acid transport and coenzyme transport and metabolism; and signal transduction and cell translation: these function in binding and transport of essential nutrients in the early stages of infection and bacterial replication. Using the VFDB database, the genes under positive selection were grouped by virulence functions consisting of adhesion, anti-phagocytosis and iron uptake systems. In Leptospira infection an initial step is adhesion at the site of abrasion or on mucous membranes. In general, several adhesion factors are responsible for attachment to a variety of cell surfaces, such as the extracellular matrix (ECM), complement factors, macrophages, 
fibroblasts, endothelium cells and kidney cells [6, 33]. Therefore, positive selection could enhance adhesion ability and assist Leptospira in spreading throughout the body during the initial stages of infection. Moreover, positive selection was found on binding sites involved in immune recognition. Protein conformational changes may be part of a strategy for anti-phagocytosis by using surface adhesion-related proteins to trap complement factors and in turn avoid opsonization and IgG deposition on the leptospiral surface membrane [34]. In this study, four genes encoding experimentally confirmed virulence factors in pathogenic Leptospira were selected to construct 3D-structure models with positive selection site mapping to investigate possible functional implications of changes to the proteins (genes $\operatorname{tly} A, h b p A, f l i N 2$ and $l p x D 1)$. Specific amino acids in each molecule were identified as being under positive selection, but the significance of these in relation to their structure and function was not investigated further. Genes related to iron uptake systems were under positive selection, including the tlyA gene encoding hemolysin A and $h b p A$ encoding the TonB-hemin binding receptor for which the structures were analyzed. These genes may provide a synergistic effect for iron acquisition from red blood cells during Leptospira infection [35]. Previously, endoflagella and protein-related LPS biosynthesis were confirmed as true virulence factors in pathogenic Leptospira [36]. In the present study, we found positive selection on genes associated with endoflagella, including fliN2 encoding for flagella motor switch protein, flgK encoding for flagella hook-associated protein, and flhB encoding for polar flagella protein. This positive selection is likely to be important for motility of Leptospira, allowing translocation, penetration and dissemination in host tissues $[37,38]$. The gene $l p x D 1$ encoding for UDP-3-O-(3-hydroxymyristoryl) glucosamine $\mathrm{N}$-acyltransferase that is involved with lipid A biosynthesis as part of the LPS structure also was subjected to positive selection. Studies have shown that alterations to the gene $l p x D$ contribute to modification in lipid A, and subsequently influence outer membrane integrity when bacteria are subjected to changing temperatures [36, 39]. Therefore, the positive selection on lpxD1 may be involved in assisting Leptospira to adapt to temperature differences in mammalian hosts. Additionally, mutations in $l p x D 1$ have been considered to be involved with kidney and liver colonization by Leptospira in an asymptomatic animal model. It also has been suggested that pathogenic Leptospira have lipid A modification strategies to evade immune response in the early stage of infection, leading to development of chronic leptospirosis in mammalian hosts [40]. The gene colA encoding for collagenase (ColA) showed evidence of both positive selection and recombination in this study. ColA has been experimentally confirmed as a virulence factor in Leptospira [41]. Positive selection in
colA might promote Leptospira invasiveness in the host body, whilst recombination of this gene might facilitate spread of advantageous mutations to other Leptospira [32, 41].

Pathogenic Leptospira have the ability to cause chronic infection by evading the host immune response [42]. Several different strategies have been described for immune evasion including biofilm formation, an increase in LPS O-antigen, and down-regulation of outer membrane proteins [43-45]. Moreover, during prolonged infections immune pressures acting on such pathogens are likely to select for advantageous genetic variation within the genome, leading to accumulation of mutations that increase survival fitness or that cause loss of function in virulence factors [46]. The balance of these genetic alterations may contribute to the strain's adaptation and establishment of a reservoir/carrier state in an animal. Our study has improved understanding of genetic adaptation in this important zoonotic pathogen and provided promising gene targets for further research. This should lead to development of better control strategies, such as improved vaccines and more accurate diagnostic tests for use in the region.

\section{Conclusions}

This study provided genomic data and characterization of Thai $L$. interrogans serovar Paidjan stain CUDO5 and L. interrogans serovar Dadas strain CUDO8 isolated from the urine of asymptomatic dogs; furthermore, comparative genomic analysis with the genomes of $97 \mathrm{~L}$. interrogans strains were undertaken and revealed an open pan-genome for the strains. Genes with established roles in acute and chronic leptospirosis were found in both Thai canine strains. Core-SNPs phylogeny confirmed their close genetic relatedness, but the $r f b$ locus analysis identified genetic variations between them that were consistent with the different serotyping results. Genes under positive selection were identified, including genes encoding factors involved in motility, temperature adaptation, and iron acquisition: these may be involved in bacterial dissemination, thermal adaptation and nutrient uptake. Further investigations into the functions and expression of these genes, including in vivo experiments are required to expand these findings.

\section{Methods}

Bacterial strains, growth conditions and DNA extraction With approval by the Chulalongkorn University Animal Care and Use Committee (CU-ACUC; Protocol number 1531075) and the Institutional Biosafety Committee (CU-VET-IBC; Protocol number 18310430), two Thai $L$. interrogans strains named CUDO5 and CUDO8 that were isolated from the urine of asymptomatic dogs in Nan province, Thailand (year 2014), were used in this 
study. The bacteria were identified to species level by partial rrs sequencing and comparative phylogeny, and were assigned as novel sequence types (ST) ST26 and ST33 respectively by multilocus sequence analysis of seven housekeeping genes (caiB, glumU, mreA, pfkB, pntA, sucA and tpiA) [19].

L. interrogans strains CUDO5 and CUDO8 that were passaged less than five times were kept as stock culture and stored at $-80^{\circ} \mathrm{C}$ before use. A $500 \mu \mathrm{L}$ volume of each thawed stock was cultured in $5 \mathrm{~mL}$ of liquid Ellinghuasen-McCullough-Johnson-Harris (EMJH) medium with 3\% rabbit serum, and incubated at $30^{\circ} \mathrm{C}$ for two weeks. Subsequently, $300 \mu \mathrm{L}$ of the cultures were spread on blood agar and incubated at $37^{\circ} \mathrm{C}$ for two days to verify lack of contamination with other bacteria by visual inspection of surface growth. To purify and collect the leptospires, the suspensions were centrifuged at $15,000 \times g$ at $4{ }^{\circ} \mathrm{C}$ for $15 \mathrm{~min}$ and then the genomic DNA was extracted from the cell-pellet using the Wizard ${ }^{\circ}$ Genomic DNA Purification Kit (Promega, USA) following the manufacturer's instructions. The quality and quantity of the DNA were measured using a spectrophotometer to obtain the A260/230 ratio (1.51 for CUDO5 and 1.75 for CUDO8) and the A260/280 ratio (1.80 for CUDO5 and 1.83 for CUDO8) (NanoDrop ${ }^{\text {mat }}$, Thermo Fisher Scientific, USA) and Qubit ${ }^{\text {mi }}$ Fluorometric Quantitation (Thermo Fisher Scientific, USA), respectively. Genomic DNA was stored at $-20^{\circ} \mathrm{C}$ before library preparation and genome sequencing.

\section{Serotyping}

Serotyping of the L. interrogans strain CUDO5 and CUDO8 was performed at the OIE National Collaborating Centre for Reference and Research on Leptospirosis, The Netherlands. A total of 43 polyclonal rabbit antisera (pAbs) raised against strains of all pathogenic and non-pathogenic Leptospira serogroups were used in the microscopic agglutination test (MAT) to determine the serogroup (Additional 5, Table 1). Subsequently, the isolates were further identified to the serovar level using monoclonal antibodies (mAbs). Strains CUDO5 and CUDO8 were subjected to serovar typing employing the whole set of mAbs derived from Leptospira serogroup Bataviae serovar Losbanos strain LT 101-69: F129C2, F129C3, F129C4, F129C6, F129C7, F129C9, F129C15, F129C18, F129C19, F129C20, F129C24, F129C25 and F129C26, and Leptospira serogroup Grippotyphosa serovar Grippotyphosa strain Moskva V, serovar Vanderhoedeni strain Kipod 179 and serovar Grippotyphosa strain Dutch M: F71C2, F71C3, F71C9, F71C13, F71C16, F71C17, F164C1, F165C1, F165C2, F165C3, F165C7, F165C8 and $\mathrm{F} 165 \mathrm{C} 12$, respectively (https://leptospira.amc.nl/ leptospirosis-reference-centre/typing-with-monoclona1-antibodies/) (Additional file 6: Tables S1 and S2).
Genome sequencing, de novo assembly and annotation DNA from $L$. interrogans strains CUDO5 and CUDO8 was subjected to whole genome sequencing and de novo assembly, as previously described [47]. Briefly, DNA libraries were prepared from genomic DNA using the Nextera XT DNA Library Preparation kit (Illumina, USA), following the manufacturer's protocol (average insert size $=375 \mathrm{bp}$ ). Genome sequencing was carried out using Illumina MiSeq with a 250 paired-end run cycles. As a consequence of the substantial genetic variation in the serovar determinant region, and to avoid bias using reference mapping, de novo genome assembly was conducted using the A5-Miseq pipeline that consisted of 5 steps including read cleaning and base error correction, contig assembly, crude scaffolding, mis-assembly correction and final scaffolding [48]. Scaffolds were oriented and ordered against the whole genome sequence of L. interrogans serovar Lai strain 56601 using ABACAS [49]. Gaps among the scaffolds were closed using the IMAGE program [50]. The draft genomes of $L$. interrogans strains CUDO5 and CUDO8 were annotated using rapid prokaryotic genome annotation (PROKKA) [51] and Rapid Annotation using Subsystem Technology (RAST) version 4.0 [52]. Prophage sequences were identified using the PHASTER web server with the default setting [53]. Clustered regularly interspaced short palindromic repeats (CRISPRs) and Cas regions were examined by CRISPRone [54]. The spacer results from CRISPRone were further analyzed for CRISPR RNA (crRNA) targets by searching against the GenBank-Phage and RefSeq-Plasmid databases using CRISPRTarget [55]. To predict overall putative virulence genes, the genomes of strains CUDO5 and CUDO8 were search against the virulence factor database (VFDB) [56]. Moreover, a list of previously confirmed virulence genes involved with acute and chronic Leptospira infections was generated based on a literature review of in vitro and in vivo experimental models [24, 36, 57] (Additional file 1: Table S7). From these, 33 representative genes with good data support were selected for gene orthologous identification using amino acid sequences retrieved from UniProt database: these sequences then were subjected to a BLAST search against all proteomic sequences of representative Leptospira strains using BLASTP analysis with an e-value threshold of 1e-06 and protein identity of more than $40 \%$ [58]. A heat map of the presence and absence of virulence genes among Leptospira strains was constructed using Clustergrammer with the default parameter [59].

Draft genome sequences of $L$. interrogans strains CUDO5 and CUDO8 were submitted to NCBI's database as Whole Genome Shotgun (WGS) sequences under the accession numbers NKYH02000001.1 and NKYG00000001.1, respectively [47]. 


\section{Average nucleotide identity (ANI) and phylogenomic analysis}

To define genome relatedness and similarities among members of the genus Leptospira, 22 representative genomes derived from 19 pathogens, two intermediate and one non-pathogen were recruited to be analyzed together with the two Thai Leptospira strains (Table 1). Average Nucleotide Identity (ANI) was calculated for each pair of Leptospira genomes by BLASTN analysis using JSpecies with the default setting [60].

To examine phylogenomic relationships and gain insights into the evolutionary history of L. interrogans, whole genome sequences for $97 \mathrm{~L}$. interrogans strain were recruited for the analysis (Additional file 3: Table S2), using the genomic sequence of the closely related species L. kirschneri serovar Cynopteri strain 3522 CT (CYN) as an outgroup. The genomes were aligned, single nucleotide polymorphisms (SNPs) in conserved regions called, recombination sites filtered, and then concatenated as core-single nucleotide polymorphisms (core-SNPs) using parsnps in the Harvest package [61]. A phylogenetic tree constructed from aligned core-SNPs sequence was generated using the maximum-likelihood (ML) method with the GTR model and 1000 bootstrap replicates by RAxML [62]. The tree was edited and visualized using EvolView [63].

\section{Comparative genome analysis}

The $97 \mathrm{~L}$. interrogans genomes were subjected to pan-genome analysis using the Bacterial Pan Genome Analysis Pipeline (BPGA) package, version 1.2 [64]. The GenBank files obtained from the NCBI annotation were used as the input file. Orthologous proteins shared among the $L$. interrogans strains were clustered using USEARCH with $50 \%$ identity cut-off. Cluster of orthologous groups (COG) analysis was used to identify core genes, accessory genes and strain-specific genes. The pan-genome and core-genome were identified using exponential growth and decay models for each newly added L. interrogans genome using the power fit law equation " $\mathrm{f}(\mathrm{x})=\mathrm{a} \times \mathrm{x}$ ". A pan-genome curve was generated to examine whether the pan-genome of $L$. interrogans is open (having an unlimited gene repertoire) or closed (having a limited maximum number of genes in the gene pool) [65]. Moreover, strain-specific genes of strain CUDO5 and CUDO8 were identified using eggNOG [66] and the NCBI database, incorporating default parameters.

\section{Positive selection on orthologous genes in $13 \mathrm{~L}$. interrogans genomes}

To detect adaptive evolution in orthologous proteins shared among 13 representative L. interrogans strains, GET_HOMOLOUGUE was used to cluster protein-coding gene alignments with $80 \%$ identity and coverage [67]. Each of the 13 representative genome sequences was manually evaluated and converted to codon sequence alignment using PAL2NAL software, version 14.0 [68]. To detect positive selection, the rates of non-synonymous and synonymous substitutions were estimated using the site-model of the CODEML program in the PAML package: this determined the viable ratio of non-synonymous to synonymous substitution rates $(d \mathrm{~N} / d \mathrm{~S}$, or $\omega)$ at substitution sites among codon alignments [69]. Model M1a, that allowed sites in which $\omega=1$ or $<1$, was compared with model M2a that allowed sites with $\omega>1$. The Likelihood Ratio Test (LRT) and the likelihood statistic $(2 \Delta \ell)$ were calculated and compared with the critical value from the $\chi^{2}$ distribution with two degrees of freedom. If LRT rejected M1 and accepted M2 with an estimated $\omega>1$ this was interpreted as evidence of positive selection. The Bayes Empirical Bayes (BEB) was employed to identify positive selection sites by calculating posterior probability (PP) in each codon using a cut-off PP > 0.95 [70]. Phipack and GENECOV were used to identify genes under positive selection with recombination [71, 72]. Multiple testing corrections were undertaken using the procedure of Benjamini and Hochberg [73]: for all genes identified as being positive for selection, $q$-values were calculated from $p$-values using the QVALUE package from $\mathrm{R}$ [74], setting a false discovery rate (FDR) of $5 \%$ to control false positive detection. A binomial test was used to estimate associations between each of the COG and VFDB categories and the frequency of positive selection.

\section{Mapping of positive selection on protein tertiary structure}

To construct protein tertiary structure, amino acid sequences deduced from genes under positive selection were modeled using homology-based protein modeling with the intensive mode in the Phyre2 (Protein Homology/analogy Recognition Engine) web server [75]. Three-dimensional (3D) protein structure was mapped with amino acid residues under positive selection and visualized by PyMOL (The PyMOL Molecular graphic System, Version 2.0 Schrödinger, LLC).

\section{Additional files}

Additional file 1: Tables S1-S9. Genomic characterization of Thai $L$. interrogans serovar Paidjan strain CUDO5 and serovar Dadas strain CUDO8. (XLSX $158 \mathrm{~kb}$ )

Additional file 2: Figure S1. The structure of the $r f b$ locus of $L$. interrogans serovar Paidjan strain CUDO5 and serovar Dadas strain CUDO8. (DOCX $4487 \mathrm{~kb})$

Additional file 3: Table S1. Average Nucleotide identity (ANI) of Leptospira strains and pan-genome analysis of $97 \mathrm{~L}$. interrogans strains. (XLSX $26 \mathrm{~kb}$ )

Additional file 4: Tables S1-S4. Statistical analysis of positive selection in 13 representative $L$. interrogans strains. (XLSX 61 kb) 
Additional file 5: Figure S1. Circular genome map of L. interrogans serovar Lai strain 56601 and the other 13 representative strains studied, with the location of 74 predicted genes under positive selection and recombination marked. (DOCX $1410 \mathrm{~kb}$ )

Additional file 6: Tables S1-S2. Leptospira strains used for serogroup and serovar typing. (XLSX $13 \mathrm{~kb}$ )

\section{Abbreviations}

ANI: Average Nucleotide Identity; BEB: Bayes Empirical Bayes; COG: Cluster of Orthologous Group; CRISPR: Clustered regularly interspaced short palindromic repeat; EMJH: Ellinghuasen-McCullough-Johnson-Harris; LRT: Likelihood Ratio Test; mAb: Monoclonal antibody; pAb: Polyclonal antibody; SNP: Single Nucleotide Polymorphism; VFDB: Virulence Factor of Bacterial Pathogen Database

\section{Acknowledgements}

AK was in receipt of awards from the 100th Anniversary Chulalongkorn University Fund for Doctoral Scholarship and the 90th Anniversary of Chulalongkorn University Fund (Ratchadaphiseksomphot Endowment Fund). We thank Dr. Pattanapon Kayansamruaj (Faculty of Fisheries, Kasetsart University, Thailand) for assistance with genomic analysis and the OIE and National Collaborating Centre for Reference and Research on Leptospirosis (Academic Medical Center, Department of Medical Microbiology, University of Amsterdam, The Netherlands) for assistance with serotyping analysis.

\section{Funding}

Not applicable.

\section{Availability of data and materials}

All data generated or analyzed in this study were obtained from the NCBI database (https://www.ncbi.nlm.nih.gov/). All genome accession numbers are listed in Table 1 of the manuscript and Additional file 3: Table S2.

\section{Authors' contributions}

AK and NV conceived and designed the study. AK performed bioinformatics analyses. AK, DJH and NV interpreted the data. CK and PS performed positive selection analysis and detailed interpretation of the data. AK, DJH and NV wrote the manuscript. The manuscript was critically revised by DJH. All authors read and approved the manuscript.

\section{Ethics approval and consent to participate}

L. interrogans serovar Paidjan strain CUDO5 and serovar Dadas strain CUDO8 were isolated from the urine of asymptomatic dogs in Nan province, Thailand (year 2014). Animal sampling and biosafety consideration in this study were approved by the Chulalongkorn University Animal Care and Use Committee (CU-ACUC; Protocol number 1531075) and the Institutional Biosafety Committee (CU-VET-IBC; Protocol number 18310430).

\section{Consent for publication}

Not applicable.

\section{Competing interests}

The authors declare that they have no competing interests.

\section{Publisher's Note}

Springer Nature remains neutral with regard to jurisdictional claims in published maps and institutional affiliations.

\footnotetext{
Author details

'Department of Microbiology, Faculty of Veterinary Science, Chulalongkorn University, Bangkok, Thailand. ${ }^{2}$ Bioinformatics and Data Management for Research Unit, Office for Research and Development, Faculty of Medicine Siriraj Hospital, Mahidol University, Bangkok, Thailand. ${ }^{3}$ Department of Infectious Diseases and Public Health, College of Veterinary Medicine and Life Sciences, City University of Hong Kong, Kowloon Tong, Hong Kong SAR. ${ }^{4}$ Diagnosis and Monitoring of Animal Pathogens Research Unit, Department of Microbiology, Faculty of Veterinary Science, Chulalongkorn University, Bangkok, Thailand.
}

Received: 19 October 2018 Accepted: 25 February 2019 Published online: 04 March 2019

\section{References}

1. Adler B, de la Pena Moctezuma A. Leptospira and leptospirosis. Vet Microbiol. 2010;140(3-4):287-96.

2. Wuthiekanun V, Sirisukkarn N, Daengsupa P, Sakaraserane P, Sangkakam A, Chierakul W, Smythe LD, Symonds ML, Dohnt MF, Slack AT, et al. Clinical diagnosis and geographic distribution of leptospirosis, Thailand. Emerg Infect Dis. 2007;13(1):124-6.

3. Evangelista K, Coburn J. Leptospira as an emerging pathogen: a review of its biology, pathogenesis and host immune responses. Future Microbiol. 2010; 5(9):1413-25.

4. Costa F, Hagan JE, Calcagno J, Kane M, Torgerson P, Martinez-Silveira MS, Stein C, Abela-Ridder B, Ko Al. Global morbidity and mortality of leptospirosis: a systematic review. PLoS Negl Trop Dis. 2015;9(9):e0003898.

5. Ren $S X$, Fu G, Jiang XG, Zeng R, Miao YG, Xu H, Zhang YX, Xiong $H$, Lu G, Lu LF, et al. Unique physiological and pathogenic features of Leptospira interrogans revealed by whole-genome sequencing. Nature. 2003;422(6934): 888-93.

6. Fouts DE, Matthias MA, Adhikarla H, Adler B, Amorim-Santos L, Berg DE, Bulach D, Buschiazzo A, Chang YF, Galloway RL, et al. What makes a bacterial species pathogenic?:comparative genomic analysis of the genus Leptospira. PLoS Negl Trop Dis. 2016;10(2):e0004403.

7. Xu Y, Zhu Y, Wang Y, Chang YF, Zhang Y, Jiang X, Zhuang X, Zhu Y, Zhang $J$, Zeng $L$, et al. Whole genome sequencing revealed host adaptationfocused genomic plasticity of pathogenic Leptospira. Sci Rep. 2016;6:20020.

8. Bulach DM, Zuerner RL, Wilson P, Seemann T, McGrath A, Cullen PA, Davis J, Johnson M, Kuczek E, Alt DP, et al. Genome reduction in Leptospira borgpetersenii reflects limited transmission potential. Proc Natl Acad Sci U S A. 2006;103(39):14560-5.

9. Toft C, Andersson SG. Evolutionary microbial genomics: insights into bacterial host adaptation. Nat Rev Genet. 2010;11(7):465-75.

10. Aguileta G, Lengelle J, Marthey S, Chiapello H, Rodolphe F, Gendrault A, Yockteng R, Vercken E, Devier B, Fontaine MC, et al. Finding candidate genes under positive selection in non-model species: examples of genes involved in host specialization in pathogens. Mol Ecol. 2010;19(2):292-306.

11. Aguileta G, Refregier G, Yockteng R, Fournier E, Giraud T. Rapidly evolving genes in pathogens: methods for detecting positive selection and examples among fungi, bacteria, viruses and protists. Infect Genet Evol. 2009;9(4):65670.

12. Yoshizaki S, Umemura T, Tanaka K, Watanabe K, Hayashi M, Muto Y. Genome-wide evidence of positive selection in Bacteroides fragilis. Comput Biol Chem. 2014;52:43-50.

13. Rojas TCG, Lobo FP, Hongo JA, Vicentini R, Verma R, Maluta RP, da Silveira WD. Genome-wide survey of genes under positive selection in avian pathogenic Escherichia coli strains. Foodborne Pathog Dis. 2017;14(5):24552.

14. Petersen L, Bollback JP, Dimmic M, Hubisz M, Nielsen R. Genes under positive selection in Escherichia coli. Genome Res. 2007;17(9):1336-43.

15. Zhang $Y$, Zhang $H$, Zhou T, Zhong $Y$, Jin Q. Genes under positive selection in Mycobacterium tuberculosis. Comput Biol Chem. 2011;35(5):319-22.

16. Soyer $Y$, Orsi RH, Rodriguez-Rivera LD, Sun Q, Wiedmann M. Genome wide evolutionary analyses reveal serotype specific patterns of positive selection in selected Salmonella serotypes. BMC Evol Biol. 2009;9:264.

17. Cao P, Guo D, Liu J, Jiang Q, Xu Z, Qu L. Genome-wide analyses reveal genes subject to positive eelection in Pasteurella multocida. Front Microbiol. 2017;8:961.

18. Makarova KS, Wolf Yl, Alkhnbashi OS, Costa F, Shah SA, Saunders SJ, Barrangou R, Brouns SJ, Charpentier E, Haft DH, et al. An updated evolutionary classification of CRISPR-Cas systems. Nat Rev Microbiol. 2015; 13(11):722-36.

19. Kurilung A, Chanchaithong P, Lugsomya K, Niyomtham W, Wuthiekanun V, Prapasarakul N. Molecular detection and isolation of pathogenic Leptospira from asymptomatic humans, domestic animals and water sources in Nan province, a rural area of Thailand. Res Vet Sci. 2017;115:146-54.

20. Ebrahimizadeh W, Rajabibazl M. Bacteriophage vehicles for phage display: biology, mechanism, and application. Curr Microbiol. 2014;69(2):109-20.

21. Decker CF, Hawkins RE, Simon GL. Infections with Pseudomonas paucimobilis. Clin Infect Dis. 1992;14(3):783-4. 
22. Majumdar S, Zhao P, Pfister NT, Compton M, Olson S, Glover CV 3rd, Wells L, Graveley BR, Terns RM, Terns MP. Three CRISPR-Cas immune effector complexes coexist in Pyrococcus furiosus. RNA. 2015;21(6):1147-58.

23. Marraffini LA, Sontheimer EJ. CRISPR interference limits horizontal gene transfer in staphylococci by targeting DNA. Science. 2008;322(5909):1843-5.

24. Picardeau M. Virulence of the zoonotic agent of leptospirosis: still terra incognita? Nat Rev Microbiol. 2017;15(5):297-307.

25. Mira A, Martin-Cuadrado AB, D'Auria G, Rodriguez-Valera F. The bacterial pan-genome:a new paradigm in microbiology. Int Microbiol. 2010;13(2):45-57.

26. Patra KP, Choudhury B, Matthias MM, Baga S, Bandyopadhya K, Vinetz JM. Comparative analysis of lipopolysaccharides of pathogenic and intermediately pathogenic Leptospira species. BMC Microbiol. 2015;15:244.

27. Riegert AS, Young NM, Watson DC, Thoden JB, Holden HM. Structure of the external aldimine form of PgIE, an aminotransferase required for $\mathrm{N}_{1} \mathrm{~N}^{\prime}$ diacetylbacillosamine biosynthesis. Protein Sci. 2015;24(10):1609-16.

28. Nascimento AL, Ko Al, Martins EA, Monteiro-Vitorello CB, Ho PL, Haake DA, Verjovski-Almeida S, Hartskeerl RA, Marques MV, Oliveira MC, et al. Comparative genomics of two Leptospira interrogans serovars reveals novel insights into physiology and pathogenesis. J Bacteriol. 2004;186(7):2164-72.

29. Corvaglia AR, Francois P, Hernandez D, Perron K, Linder P, Schrenzel J. A type III-like restriction endonuclease functions as a major barrier to horizontal gene transfer in clinical Staphylococcus aureus strains. Proc Natl Acad Sci U S A. 2010;107(26):11954-8.

30. Canchaya C, Proux C, Fournous G, Bruttin A, Brussow H. Prophage genomics. Microbiol Mol Biol Rev. 2003;67(2):238-76 table of contents.

31. Wang X, Kim Y, Ma Q, Hong SH, Pokusaeva K, Sturino JM, Wood TK. Cryptic prophages help bacteria cope with adverse environments. Nat Commun. 2010;1:147.

32. Shapiro BJ, David LA, Friedman J, Alm EJ. Looking for Darwin's footprints in the microbial world. Trends Microbiol. 2009;17(5):196-204.

33. Ko Al, Goarant C, Picardeau M. Leptospira: the dawn of the molecular genetics era for an emerging zoonotic pathogen. Nat Rev Microbiol. 2009;7(10):736-47.

34. Fraga TR, Barbosa AS, Isaac L. Leptospirosis: aspects of innate immunity, immunopathogenesis and immune evasion from the complement system. Scand J Immunol. 2011;73(5):408-19.

35. Parrow NL, Fleming RE, Minnick MF. Sequestration and scavenging of iron in infection. Infect Immun. 2013;81(10):3503-14.

36. Gomes-Solecki M, Santecchia I, Werts C. Animal models of leptospirosis: of mice and hamsters. Front Immunol. 2017:8:58.

37. Fontana C, Lambert A, Benaroudj N, Gasparini D, Gorgette O, Cachet N, Bomchil N, Picardeau M. Analysis of a spontaneous non-motile and avirulent mutant shows that FliM is required for full endoflagella assembly in Leptospira interrogans. PLoS One. 2016;11(4):e0152916.

38. Liao S, Sun A, Ojcius DM, Wu S, Zhao J, Yan J. Inactivation of the fliY gene encoding a flagellar motor switch protein attenuates mobility and virulence of Leptospira interrogans strain Lai. BMC Microbiol. 2009;9:253.

39. Li Y, Powell DA, Shaffer SA, Rasko DA, Pelletier MR, Leszyk JD, Scott AJ, Masoudi A, Goodlett DR, Wang $X$, et al. LPS remodeling is an evolved survival strategy for bacteria. Proc Natl Acad Sci U S A. 2012;109(22):871621.

40. Eshghi A, Henderson J, Trent MS, Picardeau M. Leptospira interrogans IpxD homologue is required for thermal acclimatization and virulence. Infect Immun. 2015;83(11):4314-21.

41. Kassegne K, Hu W, Ojcius DM, Sun D, Ge Y, Zhao J, Yang XF, Li L, Yan J. Identification of collagenase as a critical virulence factor for invasiveness and transmission of pathogenic Leptospira species. J Infect Dis. 2014;209(7):1105-15.

42. Monahan AM, Callanan JJ, Nally JE. Review paper: host-pathogen interactions in the kidney during chronic leptospirosis. Vet Pathol. 2009;46(5):792-9.

43. Ristow P, Bourhy P, Kerneis S, Schmitt C, Prevost MC, Lilenbaum W, Picardeau M. Biofilm formation by saprophytic and pathogenic leptospires. Microbiology. 2008;154(Pt 5):1309-17

44. Nally JE, Chow E, Fishbein MC, Blanco DR, Lovett MA. Changes in lipopolysaccharide $\mathrm{O}$ antigen distinguish acute versus chronic Leptospira interrogans infections. Infect Immun. 2005;73(6):3251-60.

45. Monahan AM, Callanan JJ, Nally JE. Proteomic analysis of Leptospira interrogans shed in urine of chronically infected hosts. Infect Immun. 2008;76(11):4952-8.
46. Dobrindt U, Zdziarski J, Salvador E, Hacker J. Bacterial genome plasticity and its impact on adaptation during persistent infection. Int J Med Microbiol. 2010;300(6):363-6.

47. Kurilung A, Keeratipusana C, Suriyaphol P, Prapasarakul N. Draft genome sequence of a Leptospira interrogans strain isolated from the urine of an asymptomatic dog in Thailand. Genome Announc. 2018;6(4):e01140-17.

48. Coil D, Jospin G, Darling AE. A5-miseq: an updated pipeline to assemble microbial genomes from Illumina MiSeq data. Bioinformatics. 2015:31(4):587-9.

49. Assefa S, Keane TM, Otto TD, Newbold C, Berriman M. ABACAS: algorithmbased automatic contiguation of assembled sequences. Bioinformatics. 2009;25(15):1968-9.

50. Swain MT, Tsai IJ, Assefa SA, Newbold C, Berriman M, Otto TD. A postassembly genome-improvement toolkit (PAGIT) to obtain annotated genomes from contigs. Nat Protoc. 2012;7(7):1260-84.

51. Seemann T. Prokka: rapid prokaryotic genome annotation. Bioinformatics. 2014:30(14):2068-9.

52. Aziz RK, Bartels D, Best AA, DeJongh M, Disz T, Edwards RA, Formsma K, Gerdes S, Glass EM, Kubal M, et al. The RAST server: rapid annotations using subsystems technology. BMC Genomics. 2008;9:75.

53. Arndt D, Grant JR, Marcu A, Sajed T, Pon A, Liang Y, Wishart DS. PHASTER: a better, faster version of the PHAST phage search tool. Nucleic Acids Res. 2016;44(W1):W16-21.

54. Zhang Q, Ye Y. Not all predicted CRISPR-Cas systems are equal: isolated cas genes and classes of CRISPR like elements. BMC Bioinformatics. 2017;18(1):92

55. Biswas A, Gagnon JN, Brouns SJ, Fineran PC, Brown CM. CRISPRTarget: bioinformatic prediction and analysis of crRNA targets. RNA Biol. 2013;10(5):817-27

56. Chen L, Zheng D, Liu B, Yang J, Jin Q. VFDB 2016: hierarchical and refined dataset for big data analysis--10 years on. Nucleic Acids Res. 2016:44(D1):D694-7.

57. Murray GL. The molecular basis of leptospiral pathogenesis. Curr Top Microbiol Immunol. 2015;387:139-86.

58. Rost B. Twilight zone of protein sequence alignments. Protein Eng. 1999;12(2):85-94.

59. Fernandez NF, Gundersen GW, Rahman A, Grimes ML, Rikova K, Hornbeck P, Ma'ayan A. Clustergrammer, a web-based heatmap visualization and analysis tool for high-dimensional biological data. Sci Data. 2017:4:170151.

60. Richter M, Rossello-Mora R, Oliver Glockner F, Peplies J. JSpeciesWS: a web server for prokaryotic species circumscription based on pairwise genome comparison. Bioinformatics. 2016;32(6):929-31.

61. Treangen TJ, Ondov BD, Koren S, Phillippy AM. The harvest suite for rapid core-genome alignment and visualization of thousands of intraspecific microbial genomes. Genome Biol. 2014;15(11):524.

62. Stamatakis A. RAxML version 8: a tool for phylogenetic analysis and postanalysis of large phylogenies. Bioinformatics. 2014;30(9):1312-3.

63. Zhang H, Gao S, Lercher MJ, Hu S, Chen WH. EvolView, an online tool for visualizing, annotating and managing phylogenetic trees. Nucleic Acids Res. 2012;40(Web Server issue):W569-72.

64. Chaudhari NM, Gupta VK, Dutta C. BPGA- an ultra-fast pan-genome analysis pipeline. Sci Rep. 2016;6:24373.

65. Tettelin H, Riley D, Cattuto C, Medini D. Comparative genomics: the bacterial pan-genome. Curr Opin Microbiol. 2008;11(5):472-7.

66. Huerta-Cepas J, Forslund K, Coelho LP, Szklarczyk D, Jensen LJ, von Mering C, Bork P. Fast genome-wide functional annotation through orthology assignment by eggNOG-mapper. Mol Biol Evol. 2017;34(8):2115-22.

67. Contreras-Moreira B, Vinuesa P. GET_HOMOLOGUES, a versatile software package for scalable and robust microbial pangenome analysis. Appl Environ Microbiol. 2013;79(24):7696-701.

68. Suyama M, Torrents D, Bork P. PAL2NAL: robust conversion of protein sequence alignments into the corresponding codon alignments. Nucleic Acids Res. 2006;34(Web Server issue):W609-12

69. Yang Z. PAML 4: phylogenetic analysis by maximum likelihood. Mol Biol Evol. 2007;24(8):1586-91

70. Yang Z, Wong WS, Nielsen R. Bayes empirical bayes inference of amino acid sites under positive selection. Mol Biol Evol. 2005;22(4):1107-18.

71. Bruen TC, Philippe $H$, Bryant D. A simple and robust statistical test for detecting the presence of recombination. Genetics. 2006;172(4):2665-81.

72. Martin D, Rybicki E. RDP: detection of recombination amongst aligned sequences. Bioinformatics. 2000;16(6):562-3. 
73. Benjamini Y, Hochberg Y. Controlling the false discovery rate - a practical and powerful approach to multiple testing. J Roy Stat Soc B Met. 1995;57(1):289-300.

74. Dabney A, Storey JD, Warnes GR: qvalue: Q-value estimation for false discovery rate control. R package version 124202010.

75. Kelley LA, Mezulis S, Yates CM, Wass MN, Sternberg MJ. The Phyre2 web portal for protein modeling, prediction and analysis. Nat Protoc. 2015;10(6):845-58.

76. Alikhan NF, Petty NK, Ben Zakour NL, Beatson SA. BLAST ring Image generator (BRIG): simple prokaryote genome comparisons. BMC Genomics. 2011;12:402.

Ready to submit your research? Choose BMC and benefit from:

- fast, convenient online submission

- thorough peer review by experienced researchers in your field

- rapid publication on acceptance

- support for research data, including large and complex data types

- gold Open Access which fosters wider collaboration and increased citations

- maximum visibility for your research: over $100 \mathrm{M}$ website views per year

At $\mathrm{BMC}$, research is always in progress.

Learn more biomedcentral.com/submissions 